\title{
POSIBLES FORZANTES DE VARIACIONES DE LARGO PLAZO DE LA PRECIPITACIÓN DE VERANO EN TUCUMÁN, ARGENTINA
}

\author{
Franco D. Medina ${ }^{1,2}$, Flavia M. Bazzano ${ }^{1,2,3}$, Teresita Heredia ${ }^{1,2}$, Ana G. Elias ${ }^{1,2}$ \\ ${ }^{1}$ Laboratorio de Física de la Atmósfera, Facultad de Ciencias Exactas y Tecnología, Universidad \\ Nacional de Tucumán, Av. Independencia 1800, 4000 Tucumán, Argentina \\ ${ }^{2}$ INFINOA (CONICET-UNT), Tucumán, Argentina \\ ${ }^{3}$ Laboratorio de Construcciones Hidráulicas, Dpto. de Construcciones y Obras Civiles, Facultad de \\ Ciencias Exactas y Tecnología, Universidad Nacional de Tucumán
}

(Manuscrito recibido el 12 de febrero de 2020, en su versión final el 9 de abril de 2020)

\section{RESUMEN}

En este trabajo se analiza la variación a largo plazo de la precipitación total y máxima diaria de verano en Tucumán, en base a datos medidos en la Estación Experimental Agroindustrial Obispo Colombres (EEAOC) durante el período 1911-2016. La precipitación total presenta tendencia lineal positiva significativa en el período completo de análisis por la presencia del salto climático de 1956. La serie de precipitación máxima diaria sólo presenta tendencia positiva significativa en el sub-periodo 1956-1994. Teniendo en cuenta que el monzón sudamericano es un fuerte determinante de los regímenes de precipitación del norte argentino, se analiza la correlación con variables de circulación asociadas: viento zonal (U) en la troposfera superior en la región de la Alta de Bolivia y viento meridional (V) en la troposfera inferior en la región de la Baja del Chaco, junto con la presión $(\mathrm{P})$ y temperatura $(\mathrm{T})$ en el Atlántico Sur. Hay buen acuerdo estadístico entre las series de precipitación y estas variables cuando se considera el período completo, porque todas presentan -en general- una tendencia lineal neta. En el sub-período 1960-2012, la precipitación total tiene una cuasi-periodicidad de 20 años en fase con P, no tiene correlación con U y V, y disminuye su correlación con T. En cambio, la precipitación máxima en este sub-período tiene buena correlación con las variables de velocidad, las cuales están ligadas a la convección regional. Se analiza también la posible asociación con algunos índices oceánicos detectándose buena correlación con la Oscilación Decadal del Pacifico (PDO)desde mediados del siglo XX. Finalmente se analiza el posible rol sobre las tendencias del aumento de $\mathrm{CO}_{2}$ global y la variación de la columna de $\mathrm{O}_{3}$ polar. Hay correlación positiva y significativa de las series de precipitación con el $\mathrm{CO}_{2}$, muy similar a la dependencia con $\mathrm{T}$, debido a que ambas series de precipitación presentan una tendencia general a aumentar. Con el $\mathrm{O}_{3}$ se observa correlación negativa significativa debido a que las series coinciden en el cambio del signo de tendencia en 1960.

Palabras clave: precipitación total, precipitación máxima diaria, circulación troposférica, forzantes antropogénicos, cambio climático.

\section{POSSIBLE LONG-TERM TREND FORCINGS OF THE SUMMER PRECIPITATION OVER TUCUMÁN, ARGENTINA}

Dirección Electrónica: medinafranco909@gmail.com 


\begin{abstract}
The long term variation of total and daily maximum precipitation over Tucuman during summer is analyzed in this work. Precipitation data cover the period 1911-2016 and was measured at the Experimental Agroindustrial Obispo Colombres (EEAOC). Total precipitation presents a significant positive linear trend during the whole period due to the 1956 climatic jump, while maximum daily precipitation presents a significant trend only during 1956-1994. Taking into account that the South American monsoon is a strong determinant of precipitation regimes in Northern of Argentina, the correlation with atmospheric circulation variables is analyzed: upper troposphere zonal wind $(\mathrm{U})$ in the Bolivian High region and lower troposphere meridional wind $(\mathrm{V})$ in the Chaco Low region, together with pressure $(\mathrm{P})$ and temperature $(\mathrm{T})$ in the South Atlantic region. There is a good statistical agreement between precipitation series and these variables when the whole period is considered due to all these series present a general linear trend. In the sub-period 1960-2012 total precipitation presents a 20-year quasi-periodicity in phase with $\mathrm{P}$, and have no correlation with $\mathrm{U}$ and $\mathrm{V}$. Maximum daily precipitation instead correlates better with velocity variables during this same period, which in turn are linked to the regional convection. The association with the oceanic variability is analyzed finding good correlation with the Pacific Decadal Oscillation (PDO) during the second half of twentieth century. Finally, the possible role on the trends of the global $\mathrm{CO}_{2}$ increase and the polar column $\mathrm{O}_{3}$ depletion is analyzed. There is a significant positive correlation with $\mathrm{CO}_{2}$, similar to that with $\mathrm{T}$, due to the general increasing trend in both series, and negative correlation with $\mathrm{O}_{3}$ due to, in addition to the opposed trends in both series, they have a coincident sign-trend change in 1960.

Keywords: total precipitation, maximum daily precipitation, tropospheric circulation, anthropogenic forcings, climate change.
\end{abstract}

\section{INTRODUCCIÓN}

La precipitación tiene gran impacto en diversos aspectos que afectan directamente a la sociedad. En particular los eventos de fuerte precipitación pueden generar inundaciones, pérdida de vidas humanas y bienes materiales, daños en los ecosistemas, y disminución de la seguridad alimentaria. Por ello es de gran interés social y económico conocer sus variaciones en distintas escalas de tiempo para una adecuada toma de decisiones tendientes a mejorar el aprovechamiento del recurso hídrico y minimizar riesgos a futuro.

Los modelos climáticos en general han predicho, junto con el aumento de temperatura global, una intensificación general del ciclo hidrológico, generando un aumento en la precipitación en la mayor parte del planeta acompañado de una disminución en algunas áreas subtropicales (Meehl et al., 2007). De esta manera, regiones con cuantiosas lluvias podrían volverse aún más lluviosas, mientras que en las zonas áridas se acentuaría la aridez (Biasutti, 2013). En particular en Argentina, se observaron tendencias positivas en la precipitación en gran parte del país durante 1916-1991, y especialmente desde la década del '50 (Barros et al., 2000a).

Las mencionadas alteraciones en el régimen pluvial son motivo de numerosos estudios recientes donde se evidencia la acción combinada de forzantes naturales y antropogénicos. Entre ellos, la concentración de ozono $\left(\mathrm{O}_{3}\right)$ en la estratosfera polar tendría notable incidencia en 
la precipitación. En este sentido, Kang et al. (2011) mostraron que las precipitaciones estivales aumentan significativamente en los subtrópicos del hemisferio sur cuando los modelos climáticos se integran con concentraciones reducidas de $\mathrm{O}_{3}$. Por otra parte, Gonzalez et al. (2014) mostraron que en 1960-1999 la disminución de la concentración de $\mathrm{O}_{3}$ produjo en el sudeste de Sudamérica un aumento de la precipitación igual o mayor que el esperable por el aumento del $\mathrm{CO}_{2}$ global.

La provincia de Tucumán $\left(\sim 26^{\circ} \mathrm{S}-28^{\circ} \mathrm{S}\right.$, $\sim 65^{\circ} \mathrm{O}-66^{\circ} \mathrm{O}$ ), Argentina, corresponde a una estrecha región húmeda que limita al oeste con un gran desierto cordillerano y al este con la llanura semiárida del Chaco. Tiene un clima subtropical con una marcada concentración de la precipitación en los meses de verano, con ocurrencia de intensos eventos diarios de precipitación superiores a $50 \mathrm{~mm}$, seguido de una estación seca en invierno. El régimen climático de verano está dominado por el sistema de circulación denominado monzón sudamericano. El mismo es inducido por las diferencias de temperatura entre el continente y los océanos, y modula la circulación atmosférica sobre la región tropical y subtropical (Vera et al., 2006; Ferrero y Villalba, 2019). Hacia fines de noviembre, durante la estación húmeda del Amazonas ocurre una intensa convección que abarca gran parte de la región central de Sudamérica desde el Ecuador hasta los $20^{\circ} \mathrm{S}$, cuya respuesta a escala regional en el verano consiste en una celda de alta presión en la troposfera superior llamada Alta de Bolivia. En la troposfera inferior aparece una baja presión denominada Baja del Chaco (Ferrero y Villalba, 2019). Esto favorece el transporte meridional de vapor desde el Amazonas hacia el sudeste de Sudamérica, en el lado este de la cordillera de los Andes, mediante un cinturón estrecho de vientos conocido como Jet de Niveles Bajos, el cual fue ampliamente estudiado por diversos autores (Campetella y Vera, 2002; Marengo et al. 2004).

Existen varios estudios previos que analizan la variabilidad de la precipitación en Tucumán.
Entre ellos, Minetti et al. (2003) observan una tendencia cuasi-lineal creciente en la precipitación anual en el período 1930-2000, con un "salto climático" en 1950, y de otro similar en 1970. Por su parte, Medina y Minetti (2004) postulan que dicho incremento de la lluvia total anual observado se asocia a un cambio en los extremos diarios de precipitación. Bazzano et al. (2019) en un estudio de las tendencias registradas en la lluvia de varias estaciones de la provincia de Tucumán observan un comportamiento variable, y un incremento no significativo de los valores máximos anuales, aunque significativo en los acumulados anuales en la estación con mayor longitud de registro. Además, se evidencian diferencias según el período considerado, con un cambio en la tendencia en las décadas recientes. De acuerdo a Minetti y Leiva (2005) entre los factores intervinientes en la variabilidad de la precipitación, se destaca la incidencia de la Oscilación Cuasi Bienal (QBO) en la lluvia de verano, que generaría la inversión del signo de las anomalías presentes en un año respecto al otro (indicando una importante oscilación de dos años), y la presencia de la Oscilación Subtropical (OST) manifiesta en fluctuaciones con 20 años de periodicidad en la lluvia de primavera y en la presión atmosférica sobre el Océano Atlántico Sur. En un trabajo anterior, Minetti (1999) también observa cierta coincidencia entre períodos de sequía y eventos intensos de La Niña, que provocaron un cambio en la tendencia creciente de la lluvia a mediados de la década de los '80, cuando había alcanzado su máximo valor.

Los resultados obtenidos en los antecedentes citados muestran la necesidad de profundizar el análisis incorporando información que permita explicar el comportamiento de la variable a largo plazo, considerando la influencia de variables climáticas y forzantes antropogénicos. Este análisis no solo sería útil para una adecuada gestión del recurso hídrico, sino que también permitiría mejorar las proyecciones basadas en modelos climáticos globales, mediante la aplicación de técnicas de downscalling estadístico (Labraga, 2010; Palomino Lemus et al., 2017; 
Bettolli y Penalba, 2018), para evaluar el comportamiento de la lluvia local futura según distintos escenarios de cambio climático.

En este trabajo se analizan las variaciones a largo plazo de la precipitación en Tucumán utilizando una serie medida en una estación meteorológica de superficie. Como posibles fuentes de estas variaciones se analizan las tendencias en las variables de circulación atmosférica asociados al sistema cuasi-monzónico sudamericano de verano y variables de presión y temperatura en el Océano Atlántico, así como también el $\mathrm{CO}_{2}$ global y el $\mathrm{O}_{3}$ polar como posibles forzantes antropogénicos de parte de las tendencias.

\section{DATOS}

Se analiza la precipitación diaria en la Estación Experimental Agroindustrial Obispo Colombres (EEAOC), ubicada en la localidad de El Colmenar $\left(26,8^{\circ} \mathrm{S}-65,2^{\circ} \mathrm{O}\right)$, en la provincia de Tucumán, Argentina. La EEAOC posee una estación meteorológica con registros continuos y controlados desde 1911. Hasta su reubicación en el aeropuerto de la provincia en 1981, la estación del Servicio Meteorológico Nacional estuvo ubicada en el predio de la EEAOC. A partir de entonces la EEAOC continuó las mediciones en la primera ubicación, dando continuidad al registro. Por lo tanto, la estación seleccionada, además de presentar un largo periodo, presenta la ventaja de no registrar cambios en su ubicación. En este trabajo se analiza la serie diaria que abarca el periodo 1911-2016, a partir de la cual se calcularon la precipitación máxima diaria para la estación verano (diciembre-enero-febrero, DJF) y para el año hidrológico considerado desde el 1 de julio al 30 de junio del año siguiente (denominando en lo sucesivo a cada año hidrológico por el segundo año que lo conforma), así como la total acumulada durante DJFy el año hidrológico. Las cuatro series obtenidas se muestran en la Figura 1. Se observa que el comportamiento de las series (totales y máximos) anuales son similares a sus análogos deDJF dado el régimen monzónico de precipitación. Esto es así a pesar de la existencia de algunos máximos anuales fuera de DJF. La similitud de las curvas polinómicas ajustadas permite asumir que la precipitación de DJF constituye una buena representación del ciclo hidrológico anual, justificándose su elección para continuar el análisis.

Teniendo en cuenta que la circulación de verano en la troposfera superior del hemisferio sur está caracterizada por tres anticiclones continentales y tres ciclones oceánicos, de los cuales interesan para nuestra región de estudio la Alta de Bolivia, las Bajas del Atlántico Sur y Pacífico Sur, y que el flujo es fundamentalmente zonal, se adopta el viento zonal a $200 \mathrm{hPa}$ como variable representativa de la circulación en la tropósfera superior (Chen et al., 1999). En cuanto a la troposfera inferior, al este de los Andes hay un importante flujo meridional de vientos (con una velocidad de $12 \mathrm{~m} \mathrm{~s}^{-1}$ en $850 \mathrm{hPa}$ a los $\left.62^{\circ} \mathrm{O}\right)$ que corresponde al Jet de Niveles Bajos (Marengo et al., 2004). Los niveles de $200 \mathrm{hPa}$ y $850 \mathrm{hPa}$ son utilizados comúnmente en estudios climáticos, como es el caso del estudio de la circulación monzónica, realizada por Gan et al. (2005) quienes analizan los campos de viento zonal y meridional junto con la precipitación en Sudamérica. A nivel superficial, como se mencionó en la introducción, hay un patrón que consiste de altas presiones en los océanos subtropicales y una baja continental.

En vista de lo expuesto anteriormente, se analizaron series de viento zonal a $200 \mathrm{hPa}$ $(\mathrm{U})$, viento meridional a $850 \mathrm{hPa}(\mathrm{V})$, presión a nivel del mar $(\mathrm{P})$, temperatura superficial $(\mathrm{T})$ y columna total de ozono $\left(\mathrm{O}_{3}\right)$ obtenidas del Reanálisis del siglo XX versión V2c (Twentieth Century Reanalysis V2c, 20CR V2c) (Compo et al., 2011) provisto por NOAA/OAR/ESRL PSD, Boulder, Colorado(disponibles en https://www esrl.noaa.gov/psd/data/20thC_Rean/). El reanalisis del siglo XX asimila observaciones de presión superficial y prescribe como condiciones de borde observaciones mensuales de temperatura superficial del mar y distribución de hielo marino. La versión V2c cubre el período 1851-2012 con una resolución espacial de 2,0 $\mathrm{x}$ 

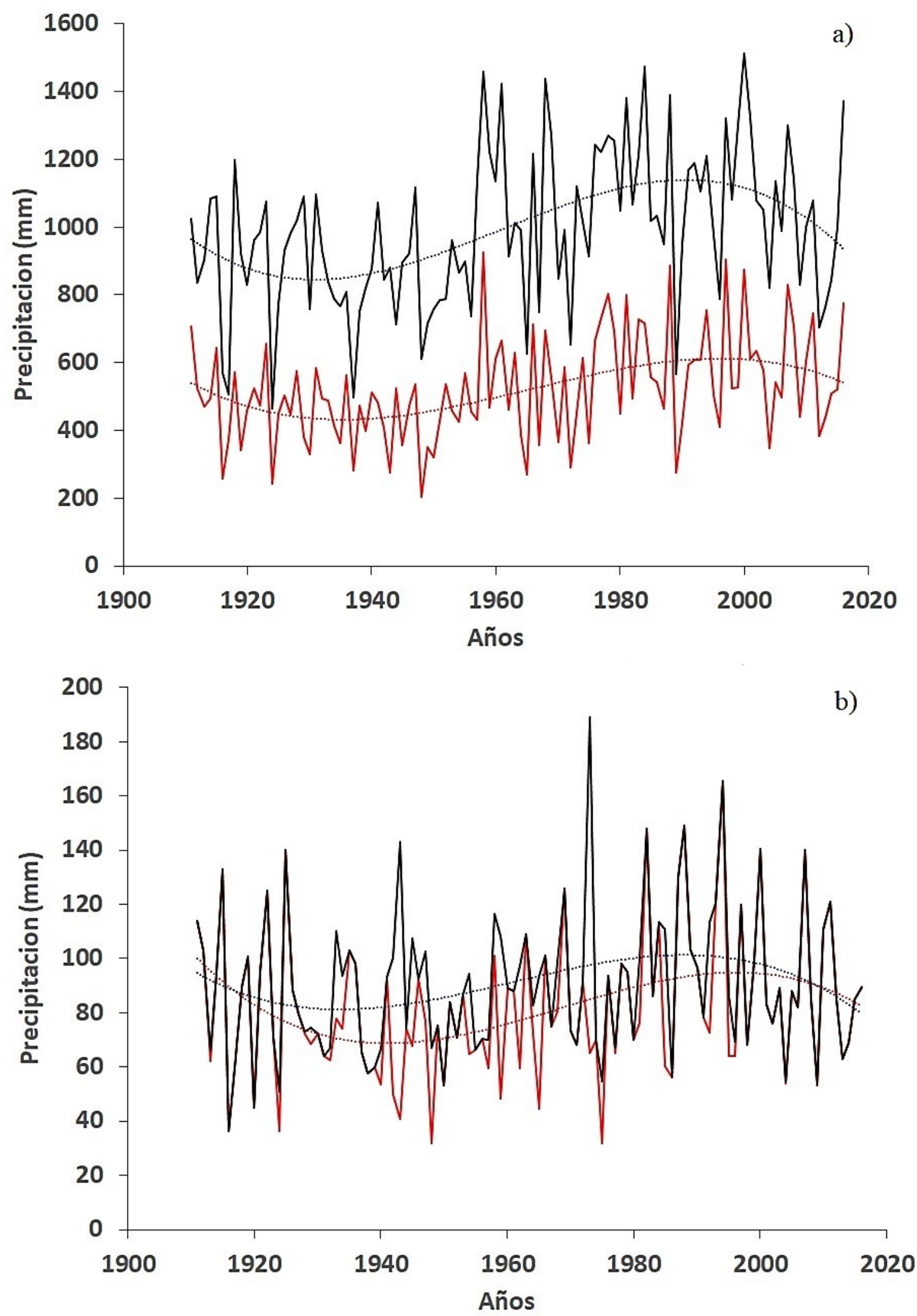

Figure 1: Series de precipitación anual (línea negra) y de verano (Diciembre-Enero-Febrero, DJF) (línea roja): a) total y b) maxima diaria. Suavizado polinómico de grado 3 (líneas de trazos). 
$2,0^{\circ}$ (lat x lon). Para el área del hemisferio Sur comprendida entre $20^{\circ} \mathrm{S}$ y $90^{\circ} \mathrm{S}$ el número de observaciones de presión entre 1900 y 1940 es de 50 (en promedio). Después de 1940 hay un sostenido aumento del número de observaciones disponibles, superando las 1000 desde el año 2000. A su vez, el error de las estimaciones de presión es el doble en la primera mitad del siglo XX, respecto al error promedio después del 2000 (Compo et al., 2011), por lo que los campos estimados para el inicio del siglo XX tienen mayor incertidumbre.

La serie de $\mathrm{O}_{3}$ polar utilizada corresponde al mes de septiembre en la región comprendida entre $60^{\circ} \mathrm{S}-90^{\circ} \mathrm{S}$. Esta elección se basa en el análisis de Polvani et al. (2011), quienes mostraron que, si bien el mínimo de $\mathrm{O}_{3}$ polar ocurre en el periodo primaveral austral, existe un retraso de dos meses para que la señal estratosférica se propague hacia la troposfera. De esta manera, el mínimo primaveral de $\mathrm{O}_{3}$ afecta al clima de DJF más que a otras estaciones del año.

Además, se analiza la serie anual de concentración global de dióxido de carbono $\left(\mathrm{CO}_{2}\right)$ del Institute for Atmospheric and Climate Science (IAC) (disponible en https: //www.co2.earth/historical-co2-datasets) para el periodo 1911-2012.

\section{METODOLOGÍA}

Como primer paso se estimaron las tendencias lineales de las series de precipitación de DJF a través del estimador no paramétrico de Sen (Sen, 1968). La significancia estadística se evaluó con el test de Mann-Kendall de uso común en el análisis de tendencias en series de tiempo climáticas e hidrológicas (Hamed, 2008). Se utilizó el test de Pettitt (Pettitt, 1979) como método de detección de punto de cambios, ya sea por alteraciones en la media, varianza y/o en la amplitud. Para llevar a cabo esto se utilizó el paquete "trend" (ThorstenPohlert, 2018) del software libre R versión 3-6-1 (R Core Team, 2019). Se analizaron el periodo 1911-2016 y el sub-periodo 1956-1994. Este último se eligió en base al salto climático de 1956 (Minetti y Vargas, 1997) y a que en la Figura 1a se observa "a ojo desnudo" una pendiente positiva a partir de ese año, aproximadamente, hasta 1994. Por otro lado, en la Figura 1-b de la máxima diaria hay tendencia positiva desde 1956 hasta 1994 cuando ocurre un pico superior a $160 \mathrm{~mm}$. El objetivo fue evaluar la magnitud y la significancia de la pendiente en ese sub-periodo en ambas series, para determinar en qué medida la tendencia de la máxima diaria está presente en la total de verano.

Se evaluó luego la correlación espacial entre las series de precipitación de verano de Tucumán $\mathrm{y}$ las series de V, U, P y T de DJF en la región comprendida entre $10^{\circ} \mathrm{N}-90^{\circ} \mathrm{S}$ y $20^{\circ} \mathrm{O}$ - $100^{\circ} \mathrm{O}$ que abarca Sudamérica y la zona Sur de los Océanos Atlántico y Pacífico, utilizando la aplicación Explorador Climático KNMI (KNMI Climate Explorer: https://climexp knmi.nl/) del Real Instituto Meteorológico de los Países Bajos (Koninklijk Nederlands Meteorologisch Instituut, KNMI), que forma parte de la Organización Meteorológica Mundial (WMO, World Meteorologial Organization). Esta aplicación contiene datos climáticos y herramientas de análisis estadístico (temporal y espacial), y genera resultados numéricos y gráficos (Van Oldenborgh et al., 2008; Trouet y Van Oldenborgh, 2013).

Finalmente, se analizó la asociación de las tendencias a largo plazo de las series de precipitación con las series de V, U, P y $\mathrm{T}$ promediadas en determinadas regiones (recuadros en las Figuras 3 a, c, e y g, respectivamente). La elección de estas regiones se hizo en base al coeficiente de correlación y a la posible conexión de las mismas con el monzón sudamericano. La significancia estadística se estimó utilizando el test t de Student. Para evaluar la influencia de la variabilidad oceánica, se correlacionaron las series de precipitación, U, V y T con las series del Índice de Oscilación del Sur (SOI, Southern Oscillation Index) (https://crudata.uea.ac.uk/cru/data/soi/ soi.dat), Oscilación Decadal del Pacifico (PDO, 


\begin{tabular}{|c|c|c|c|c|}
\hline \multirow{2}{*}{ Serie de precipitación } & \multicolumn{2}{|c|}{$1911-2016$} & \multicolumn{2}{c|}{$1956-1994$} \\
\cline { 2 - 5 } & $\begin{array}{c}\text { Pendiente de } \\
\text { Sen [mm/año] }\end{array}$ & $\mathrm{p}$ & $\begin{array}{c}\text { Pendiente de } \\
\text { Sen [mm/año] }\end{array}$ & $\mathrm{p}$ \\
\hline Máxima diaria (anual) & 0,11 & 0,22 & 0,62 & 0,09 \\
\hline Máxima diaria (DJF) & 0,15 & 0,08 & $\mathbf{0 , 8 5}$ & 0,03 \\
\hline Total (anual) & $\mathbf{2 , 6 6}$ & 0,0005 & 1,34 & 0,70 \\
\hline Total (DJF) & $\mathbf{1 , 6 1}$ & 0,001 & 3,34 & 0,26 \\
\hline
\end{tabular}

Table I: Pendiente de Sen y significancia de acuerdo al test de Mann-Kendall para la precipitación total y máxima diaria anual y de la estación de verano en Tucumán, para el período 1911-2016 y sub-período 1956-1994. Se remarcan en negrita los valores con nivel de confianza mayor al $95 \%$ $(p<0,05)$.

Pacific Decadal Oscillation) (http://research jisao.washington.edu/pdo/PDO.latest.txt) y Oscilación Multidecadal del Atlántico (AMO, Atlantic Multidecadal Oscillation) (https://www.esrl.noaa.gov/psd/data/ correlation/amon.us.long.data). En el caso del SOI, siguiendo la metodología de Barreiro et al. (2014), se filtró la influencia de la PDO mediante una regresión lineal. Se calculó la correlación con un promedio móvil de 5 años para filtrar las altas frecuencias. Lo mismo se hizo con las series de $\mathrm{O}_{3}$ polar y $\mathrm{CO}_{2}$ global para evaluar la influencia antropogénica.

En el análisis de correlación se analizaron los periodos 1911-2012 y el sub-periodo 1960-2012. La elección de este último se fundamenta en el cambio de signo observado en la pendiente de la serie de $\mathrm{O}_{3}$ aquí analizada en 1960 y al aumento de la pendiente de la serie de $\mathrm{CO}_{2}$. Con esto se busca evaluar la existencia de cambios cuando se analiza el periodo en que parece variar la tendencia de los forzantes antropogénicos.

\section{RESULTADOS}

\subsection{Tendencias de largo plazo}

La Tabla I muestra las pendientes de las series de precipitación de Tucumán. Hay tendencias positivas significativas con nivel de confianza mayor a $95 \%$ en las series de precipitación total y al $92 \%$ para la máxima de verano.

Mediante el test de Pettitt, se encuentra que las series de precipitación total tienen un punto de cambio significativo en 1956, correspondiente al salto climático detectado por Minetti y Vargas (1997).

Las series de precipitación máxima diaria no presentan punto de cambio significativo según el test de Pettitt. Sin embargo, en la Figura 1 se observa un sub-período, 1956-1994, de tendencia positiva más marcada en ambos casos, total y máxima diaria, cuyos valores se listan también en la Tabla I. La pendiente de dicho sub-periodo es significativa al $95 \%$ para la serie máxima diaria de verano y al $91 \%$ para la máxima anual. La menor significancia para la máxima anual se debe a la presencia de algunos picos pronunciados no presentes en DJF (por ejemplo, en 1978) que aumentan el ruido de la serie. Respecto a esto, si bien el $72 \%$ de los máximos de precipitación anuales ocurren en verano, existen algunas diferencias en las series (Figura 1b). En la Figura 2 se muestran el mes de ocurrencia del máximo en cada año hidrológico, donde se observa que entre 1930 y 1970 hay 14 años en los cuales la máxima diaria se dio fuera de DJF. De esos 14 años, en 9 la máxima diaria ocurrió en marzo y 1 en abril, por lo que podríamos decir que en esos años hubo corrimiento de la máxima a meses posteriores a DJF. En los 4 años restantes las máximas ocurrieron en noviembre. En tanto, entre 1970 y 2012 el número de años en los que la máxima ocurrió fuera de DJF disminuyó a 9, siendo 6 casos en marzo, 2 en abril y 1 en mayo. En este último periodo no 


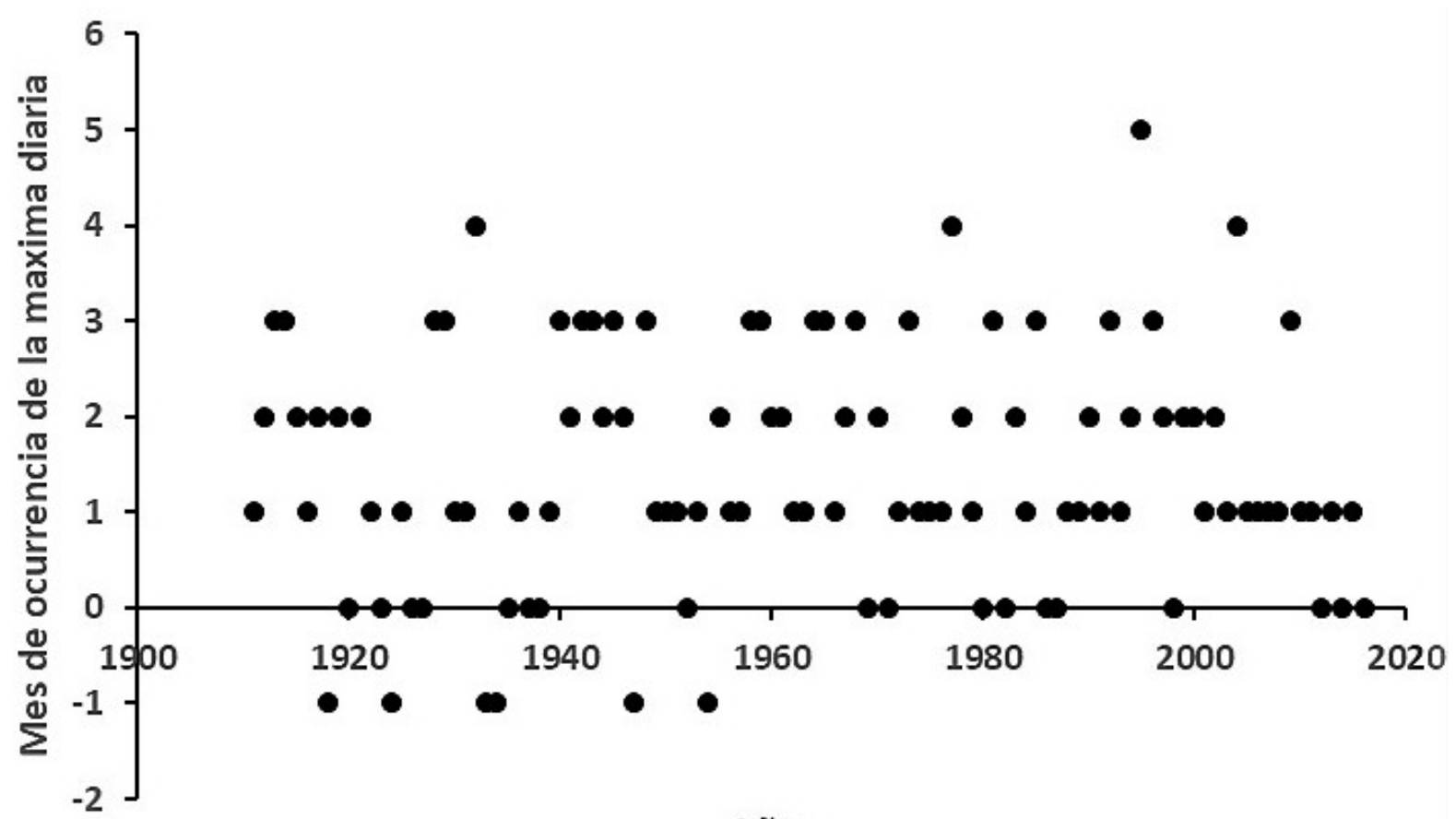

Años

Figure 2: Mes de ocurrencia de la máxima precipitación diaria para cada año. Los meses corresponden a: $-1=$ noviembre, $0=$ diciembre, $1=$ enero, $2=$ febrero, $3=$ marzo, $4=$ abril, 5 $=$ mayo.

\begin{tabular}{|c|c|c|c|c|}
\hline \multirow{2}{*}{ Variable / Período } & \multicolumn{4}{|c|}{ Variable / Región promediada } \\
\cline { 2 - 5 } & $\begin{array}{c}\mathrm{U}(200 \mathrm{hPa}) \\
/ 15-25^{\circ} \mathrm{S}, \\
60-90^{\circ} \mathrm{O}\end{array}$ & $\begin{array}{c}\mathrm{V}(850 \mathrm{hPa}) \\
/ 15-25^{\circ} \mathrm{S}, \\
50-70^{\circ} \mathrm{O}\end{array}$ & $\begin{array}{c}\mathrm{P} \\
/ 10-30^{\circ} \mathrm{S}, \\
0-20^{\circ} \mathrm{O}\end{array}$ & $\begin{array}{c}\mathrm{T} \\
/ 20-50^{\circ} \mathrm{S}, \\
30-50^{\circ} \mathrm{O}\end{array}$ \\
\hline $\begin{array}{c}\text { Precipitación total DJF / } \\
1911-2012\end{array}$ & $\mathbf{0 , 7 1}$ & $\mathbf{0 , 7 0}$ & $\mathbf{0 , 7 0}$ & $\mathbf{0 , 7 6}$ \\
\hline $\begin{array}{c}\text { Precipitación total DJF / } \\
1960-2012\end{array}$ & 0,22 & 0,17 & $\mathbf{0 , 7 2}$ & 0,44 \\
\hline $\begin{array}{c}\text { Precipitación máxima DJF / } \\
1911-2012\end{array}$ & $\mathbf{0 , 5 7}$ & $\mathbf{0 , 5 8}$ & $\mathbf{0 , 3 9}$ & $\mathbf{0 , 5 9}$ \\
\hline $\begin{array}{c}\text { Precipitación máxima DJF / } \\
1960-2012\end{array}$ & $\mathbf{0 , 5 0}$ & $\mathbf{0 , 5 5}$ & 0,30 & $\mathbf{0 , 5 7}$ \\
\hline
\end{tabular}

Table II: Coeficientes de correlación (r) entre las series de precipitación de Tucumán y las variables troposféricas U, V, P y T, para los periodos 1911-2012 y 1960-2012, a las que previamente se filtraron oscilaciones de alta frecuencia mediante un promedio móvil de 5 años. En negrita se marcan los coeficientes significativos al $95 \%$ según el test t de Student.

ocurrieron máximos previos a DJF. Por lo tanto, en las últimas décadas la máxima diaria ocurrió entre los meses de diciembre y mayo en cada año hidrológico, a diferencia del resto de la serie donde se observa que los máximos ocurren entre noviembre y abril, por lo que podríamos decir que hubo un "leve corrimiento" hacia el otoño en el periodo anual de mayores precipitaciones. 
a)

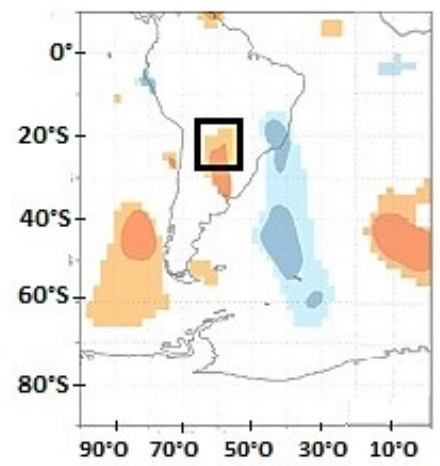

c)

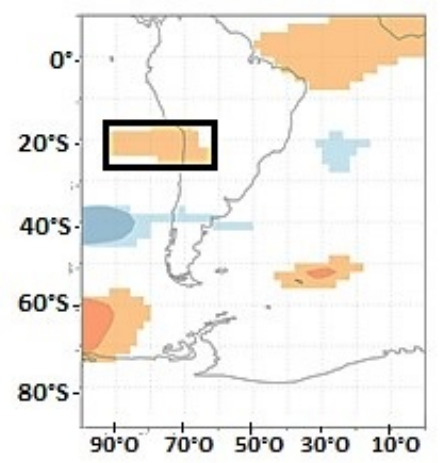

e)

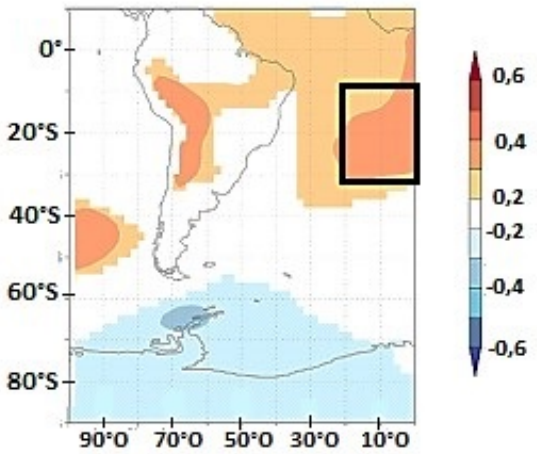

g)
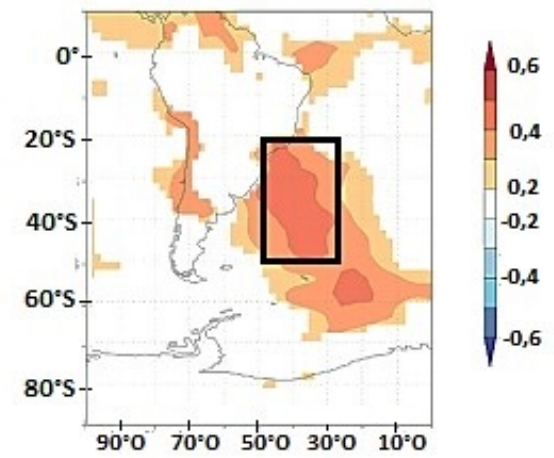

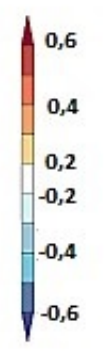

b)
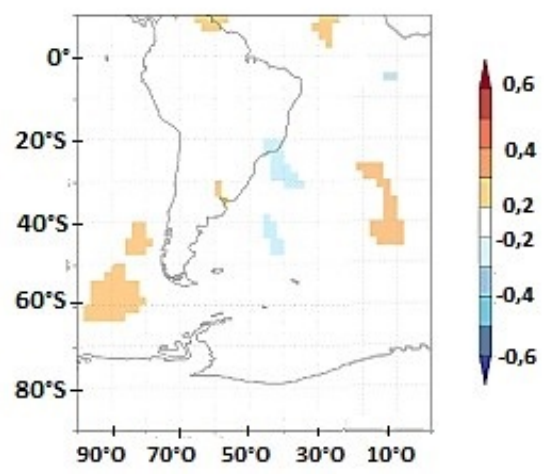

d)
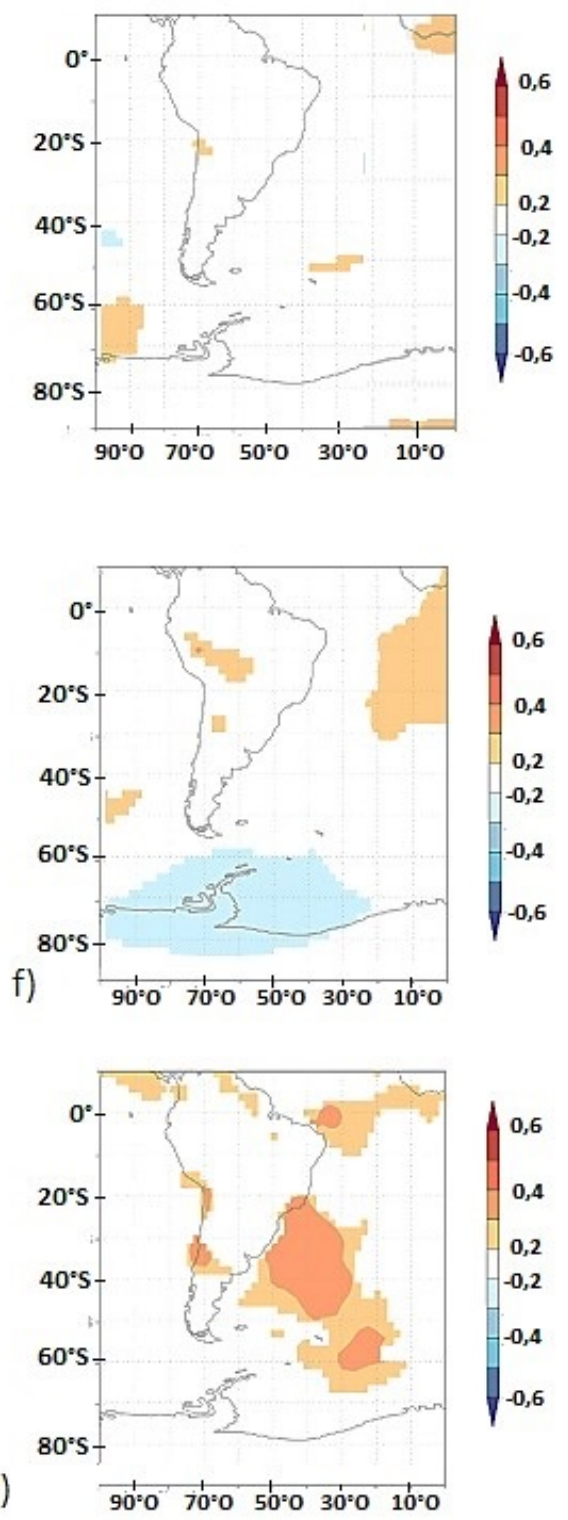

Figure 3: Mapas de correlación de: a) y b) V (viento meridional a $850 \mathrm{hPa}$ ), c) y d) U (viento zonal a $200 \mathrm{hPa}$ ), e) y f) P (presión a nivel del mar), g) y h) T (temperatura media del aire de 2 a $10 \mathrm{~m}$ ), con las series de precipitación total (columna hacia la izquierda) y máxima diaria (columna hacia derecha) de DJF, de Tucumán. Zonas con color corresponden valores de $\mathrm{r}$ con nivel de confianza mayor a $95 \%$. Figura obtenida con el Explorador Climático KNMI. Los recuadros negros indican las regiones promediadas para V, U, P y T para un posterior análisis. 


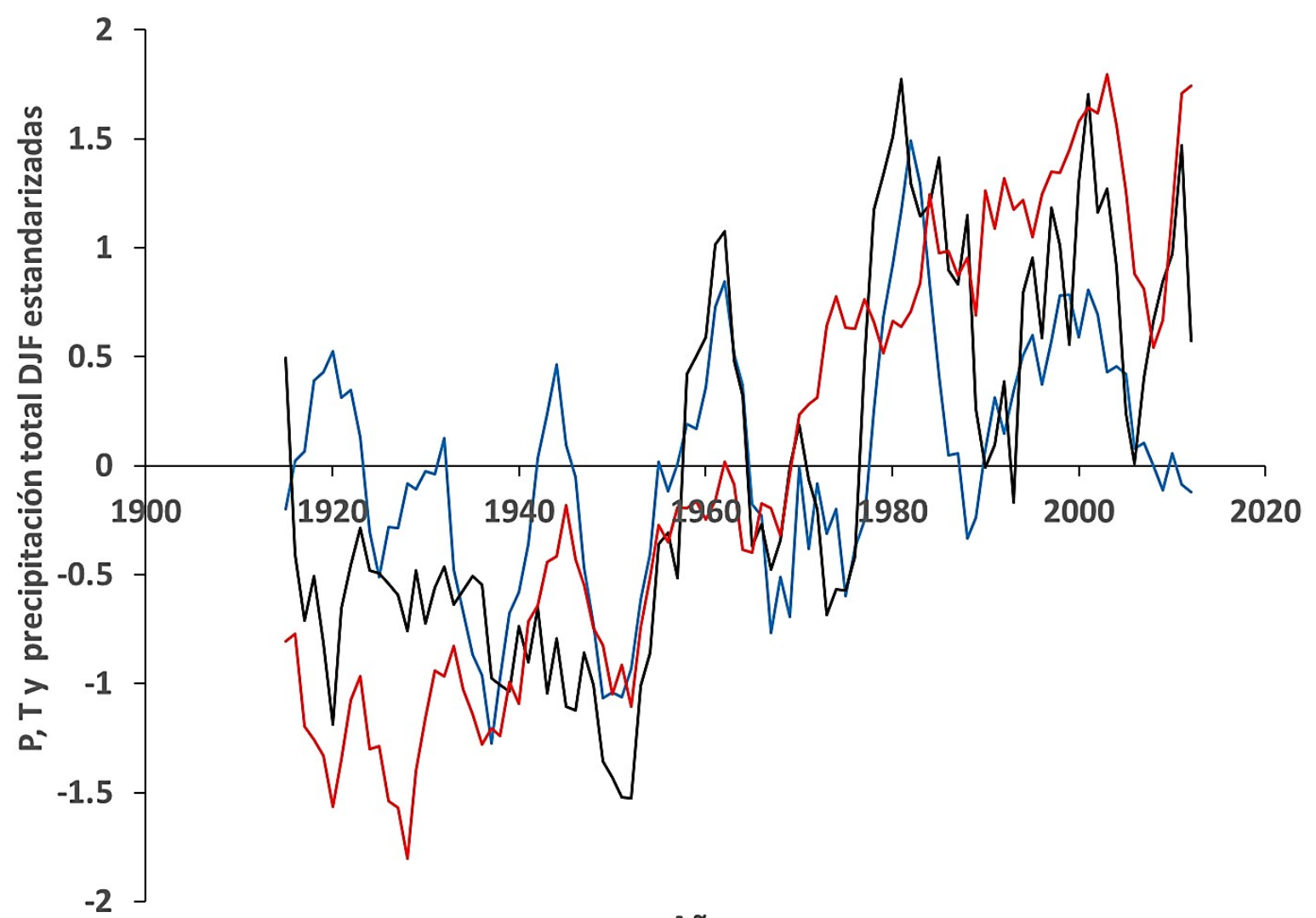

Años

Figure 4: Series de precipitación total de Tucumán (línea negra), P promedio del área comprendida entre $10^{\circ} \mathrm{S}-30^{\circ} \mathrm{S}$ y $0^{\circ} \mathrm{O}-20^{\circ} \mathrm{O}$ (línea azul) y $\mathrm{T}$ promedio del área comprendida entre $20^{\circ} \mathrm{S}-50^{\circ} \mathrm{S}$ y $30^{\circ} \mathrm{O}-50^{\circ} \mathrm{O}$ (línea roja), correspondientes a la estación de verano, DJF. Series estandarizadas y suavizadas con promedio móvil de 5 años.

En cuanto a las series de precipitación total, no presentan pendiente significativa en el sub-periodo analizado posterior al salto brusco ocurrido en 1956.

\subsection{Asociación con variables de vientos, presión y temperatura}

En la Figura 3 se muestran mapas de coeficiente de correlación (r) de las series de precipitación de Tucumán DJF y las series de V, U, P y T. Todos los valores graficados tienen nivel de confianza mayor a $95 \%$.

Hay correlación positiva entre $\mathrm{V}$ y la precipitación total (Figura 3a) en la zona del Pacifico entre los $40^{\circ}$ y $60^{\circ} \mathrm{S}$ y en el continente en la zona de la baja del Chaco, y negativa en una franja del Atlántico que sube hasta Brasil donde se ubica la Zona de Convergencia del Atlántico Sur. El mismo patrón de correlación, pero debilitado, se observa para la precipitación máxima (Figura 3b). En el caso de U y la precipitación total se observa correlación positiva con la zona de la Alta de Bolivia, el Atlántico ecuatorial, la zona sur de los océanos Atlántico y Pacífico. Se observa correlación negativa con una zona del Pacífico entre los $35^{\circ} \mathrm{S}$ y $45^{\circ} \mathrm{S}$. En el caso de la precipitación máxima diaria este patrón se ve casi desdibujado, excepto la correlación con las zonas sur de ambos océanos.

En el caso de P y la precipitación total (Figura 3e) se observa una correlación positiva con valores de $r$ entre 0,3 y 0,4 en la zona del Atlántico a la 


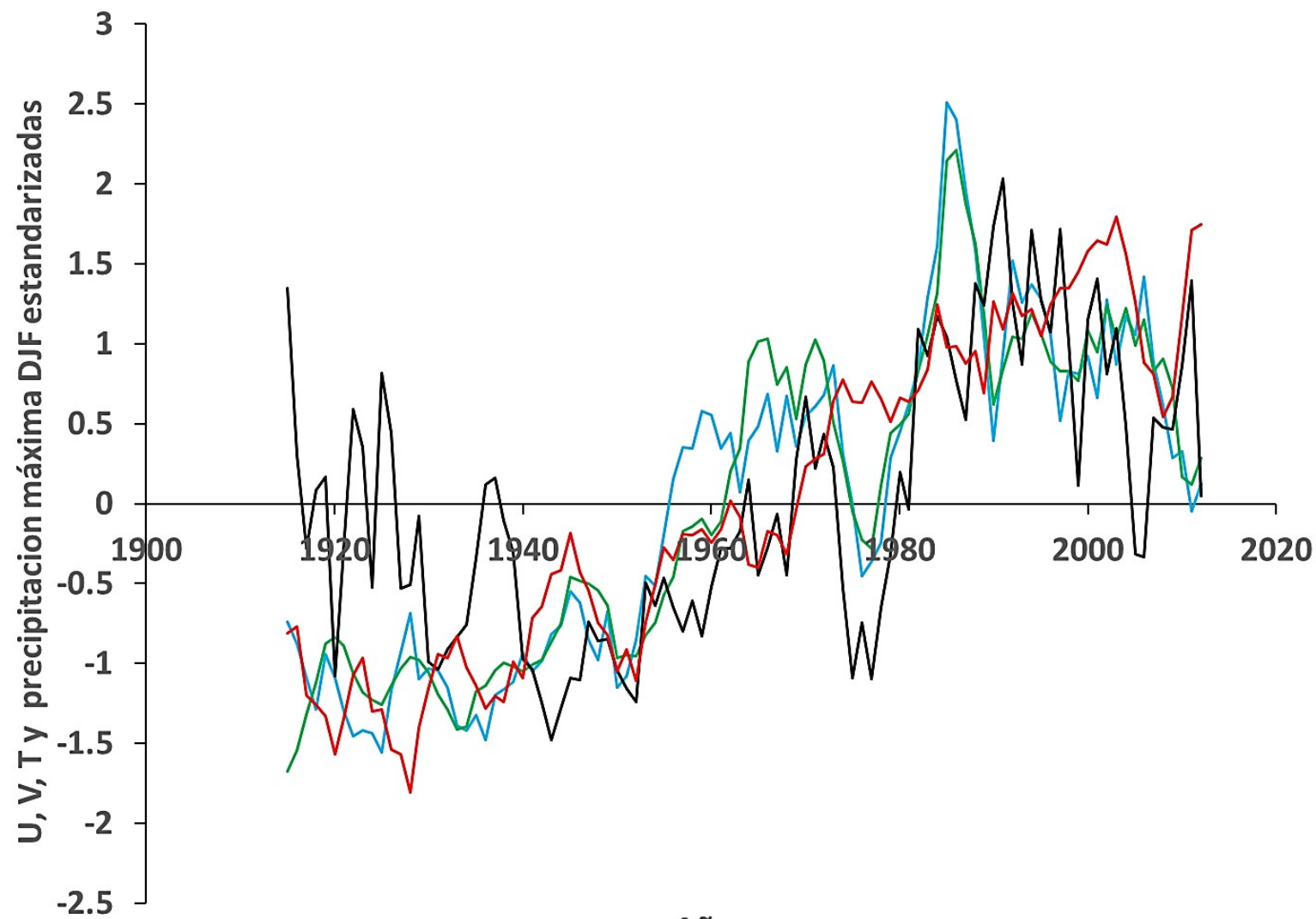

Figure 5: Series de precipitación máxima diaria de Tucumán (línea negra), U promedio del área comprendida entre $15^{\circ} \mathrm{S}-25^{\circ} \mathrm{S}$ y $60^{\circ} \mathrm{O}-90^{\circ} \mathrm{O}$ (línea celeste), $\mathrm{V}$ promedio del área comprendida entre $15^{\circ} \mathrm{S}-25^{\circ} \mathrm{S}$ y $50^{\circ} \mathrm{O}-70^{\circ} \mathrm{O}$, (línea verde) y $\mathrm{T}$ promedio del área comprendida entre $20^{\circ} \mathrm{S}-50^{\circ} \mathrm{S}$ y $30^{\circ} \mathrm{O}-50^{\circ} \mathrm{O}$ (línea roja), correspondientes a la estación de verano, DJF. Series estandarizadas y suavizadas con promedio móvil de 5 años.

altura de Brasil, en la zona continental en Bolivia y región de Chaco y en una pequeña zona del Pacífico entre los $40^{\circ}$ y $50^{\circ}$ S. Se observa también correlación negativa con la zona Antártica. Este mismo patrón, aunque debilitado, aparece en el caso análogo con la precipitación máxima diaria (Figura 3f). En el caso de T y ambas series de precipitación hay correlación positiva con la temperatura en el Atlántico, hacia el Sur de $20^{\circ} \mathrm{S}$, y en menor medida con la de la zona de la costa del Pacífico en el centro de Chile.

Se calcularon las series promedio en las regiones de recuadros en Figura 3 (como se mencionó en la sección 3) y se estimó la correlación lineal entre ellas y cada serie de precipitación. La Tabla
II muestra los valores de $\mathrm{r}$ según la variable correspondiente y la región a la que pertenece U (Alta de Bolivia), V (jet de niveles bajos), P y T (ambas del Atlántico Subtropical). Las Figuras 4 y 5 muestran la precipitación total y la máxima diaria de DJF, respectivamente, junto a las series con que mejor correlacionan $(\mathrm{P}$ y $\mathrm{T}$ para la primera, U y T para la segunda). Todas las variables, excepto $\mathrm{P}$, parecen presentar una tendencia más monótona en el período completo. La Tabla II muestra también la correlación para el sub-período 1960-2012.

Se observa que la correlación entre los valores de precipitación y de las otras variables es positiva en todos los casos. En el caso de V, el mismo tiene 
valores negativos en la región considerada por tener sentido hacia el sur (el sentido positivo es hacia el norte por convención). Así, la correlación positiva entre $\mathrm{V}$ y la precipitación indica que en periodos en que el viento meridional $\mathrm{V}$ fue de menor intensidad (menos negativo), la precipitación fue mayor. Esto descartaría la posibilidad de que el comportamiento a largo plazo en la precipitación se explique por un aumento de la intensidad del jet de capas bajas, a diferencia de lo que ocurre en las llanuras del este de Argentina en los denominamos "eventos de jet de niveles bajos" (Nicolini et al., 2002). En los demás casos (U, P y T), la correlación positiva obtenida sugiere que el incremento en la precipitación estaría vinculado al Alta de Bolivia (aumento de U) y al Atlántico (aumento de $\mathrm{P}$ y $\mathrm{T})$.

La correlación de la precipitación total con $\mathrm{P}$ es alta en 1911-2012 y 1960-2012. A partir de 1960 ambas series evidencian una periodicidad casi en fase del orden de 20 años (Figura 3). Esta periodicidad corresponde a un patrón de variabilidad océano-continente denominado Oscilación Subtropical (OST) por Minetti y Leiva (2005) con impacto en el inicio de la estación lluviosa (noviembre-diciembre). Ellos postulan que en los meses posteriores (enero-febrero-marzo) la precipitación es principalmente afectada por factores continentales regionales y no tanto por la OST. Por otra parte, la periodicidad hallada podría estar relacionada con la periodicidad en el movimiento del baricentro solar (Cionco y Pavlov, 2018), ya analizada en conexión con la precipitación total de Tucumán por Heredia et al. (2019). El baricentro afectaría la radiación solar, que tendría a su vez impacto en el clima mediante mecanismos dinámicos objeto de gran discusión y controversia que no son objeto de este estudio. Heredia et al. (2019) argumentan la posibilidad de que este ciclo de 20 años afecte más a las características de la superficie del mar, de manera que sería esperable esta oscilación en P (Figura 4). Sin embargo, $\mathrm{T}$ también corresponde al promedio de un área íntegramente en el Atlántico, pero no evidencia esta ciclicidad. Heredia et al. (2019), quienes analizan T de Tucumán (es decir continental), encuentran una correlación lineal con parámetros del baricentro solar del orden 0,1 mientras que en el caso de la precipitación total es de 0,4. En general podríamos decir que cuando se considera el período completo 1911-2012, la tendencia lineal de largo plazo predomina sobre la variación bidecadal en el patrón de variabilidad de la precipitación total, $\mathrm{y}$ por esto la correlación con $\mathrm{T}$ es ligeramente mayor que con $\mathrm{P}$.

Los valores del coeficiente de correlación de la máxima diaria con $\mathrm{U}, \mathrm{V}$ y $\mathrm{T}$ se mantienen similares en los dos periodos analizados (Tabla II). Con P la correlación es variable según el período, y pierde significancia en 1960-2012 por la ausencia de la variación bidecadal en la máxima diaria. Como se puede observar en la Figura 5 el comportamiento a largo plazo de la precipitación máxima diaria en Tucumán fue similar al comportamiento de las series de temperaturas en el Atlántico subtropical y del viento zonal continental en niveles altos.Sin embargo, se encuentran ciertos desfasajes en las variaciones de menor amplitud. Ambos resultados estarían de acuerdo con la hipótesis de que una intensificación de la Alta de Bolivia y mayores temperaturas en el océano Atlántico estarían asociadas con más lluvia en la región. Por otra parte, se debe tener en cuenta que un análisis estadístico, sin el acompañamiento del estudio físico que explique una asociación o causa-consecuencia, no permite discriminar si las variaciones similares observadas corresponden a una relación física real existente entre las variables o si son consecuencia simplemente de una fuente externa común a ambas variables.

Las variables $\mathrm{U}, \mathrm{V}, \mathrm{P}$ y $\mathrm{T}$ no son todas independientes entre sí. La correlación entre $\mathrm{U}$ y $\mathrm{V}$ es de 0,7 , tal como se esperaría, ya que las áreas promediadas corresponden a un mismo sistema integrado por la Alta de Bolivia, la Baja del Chaco y el jet de niveles bajos. Por otra parte, la correlación de $\mathrm{P}$ con $\mathrm{U}, \mathrm{V}$ y $\mathrm{T}$ es baja $(\mathrm{r}<0,5$ en todos 


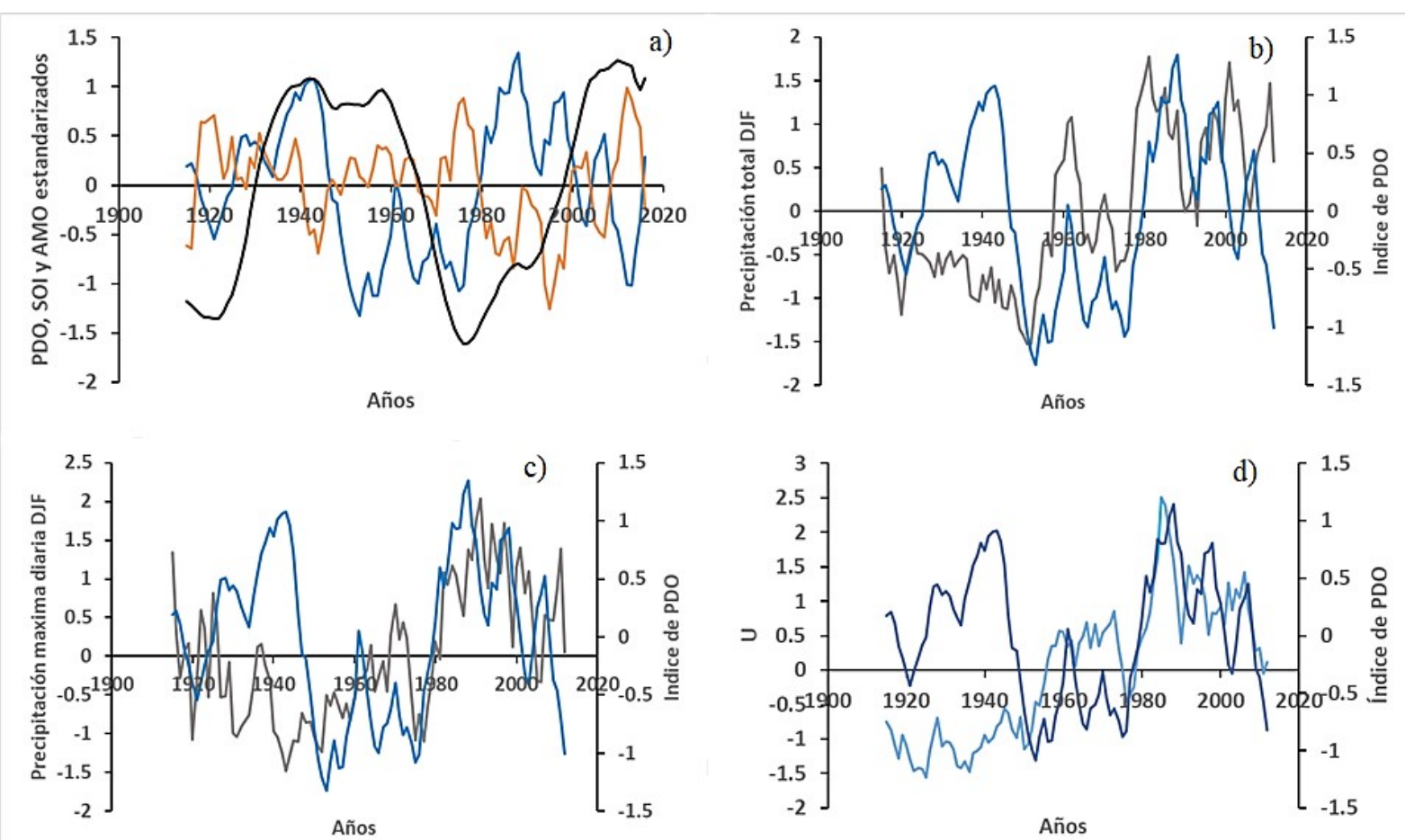

Figure 6: Series estandarizadas y con promedio móvil de 5 años de: a) PDO (línea azul), AMO (línea negra) y SOI (línea naranja), b) precipitación total de DJF (línea gris) y PDO (línea azul), c) precipitación máxima diaria de DJF (línea gris) y PDO (línea azul), d) U (línea celeste) y de PDO (línea azul).

los casos) y no significativa estadísticamente, por lo que $\mathrm{P}$ sería independiente de ellas. Físicamente podría pensarse que $\mathrm{P}$ evidenció cambios relacionados a la expansión de la Celda de Hadley (Lucas et al., 2014), los cuales en U y $\mathrm{V}$ podrían ser menores comparados con aquellos cambios que surgen de otros factores, tales como los relacionados aprocesos continentales. En relación a esto último, Vera et al. (2019) analizan la relación entre $\mathrm{U}$ en la región $15^{\circ} \mathrm{S}-20^{\circ} \mathrm{S}$ y la expansión de la Celda de Hadley global, y encuentran que no hay correlación estadísticamente significativa, postulando que la circulación podría ser influenciada por otros mecanismos independientes de la expansión tropical, tales como los relacionados al Monzón Sudamericano. La baja correlación de $\mathrm{P}$ con $\mathrm{U}, \mathrm{V}$ y $\mathrm{T}$ se debe a que $\mathrm{P}$ presenta una cuasi-periodicidad de 20 años, la cual está ausente (o es muy débil) en las otras variables.

\subsection{Asociación con variabilidad oceánica}

La Figura 6a muestra las series de PDO, AMO y SOI. No se encontraron correlaciones estadísticamente significativas entre las series oceánicas y la precipitación para el periodo 1911-2012 en ningún caso (Tabla III). En el sub-periodo 1960-2012 solo se encuentra correlación significativa con PDO, siendo $\mathrm{r}=$ 0,5 para la precipitación total y $\mathrm{r}=0,6$ para la máxima diaria. En la Figura 6 se muestran las series de precipitación total (6b) y máxima diaria (6c) con la serie de PDO. Se observa que después de 1950 las series de precipitación siguen a la serie de PDO en las variaciones de mayor magnitud (por ejemplo, en el rápido incremento entre 1975 y 1980, Figuras 6b y 6c) dando la apariencia de una modulación de la precipitación por parte de PDO. Sin embargo, hay variaciones de menor amplitud que no son compartidas y también algunos desfasajes entre las series. 


\begin{tabular}{|l|c|c|c|c|c|c|c|c|c|c|}
\hline \multirow{2}{*}{ Variables } & \multicolumn{2}{|c|}{$\mathrm{PDO}$} & \multicolumn{2}{c|}{$\mathrm{AMO}$} & \multicolumn{2}{c|}{$\mathrm{SOI}$} & \multicolumn{2}{c|}{$\mathrm{CO}_{2}$} & \multicolumn{2}{c|}{$\mathrm{O}_{3}$} \\
\cline { 2 - 11 } & $1911-$ & $1960-$ & $1911-$ & $1960-$ & $1911-$ & $1960-$ & $1911-$ & $1960-$ & $1911-$ & $1960-$ \\
& 2012 & 2012 & 2012 & 2012 & 2012 & 2012 & 2012 & 2012 & 2012 & 2012 \\
\hline $\mathbf{P}$ & 0,24 & 0,47 & $-0,25$ & 0,04 & $-0,12$ & $-0,04$ & $\mathbf{0 , 3 8}$ & 0,15 & $\mathbf{- 0 , 3 6}$ & $-0,31$ \\
\hline $\mathrm{T}$ & 0,09 & 0,45 & $-0,02$ & 0,07 & $-0,27$ & 0,28 & $\mathbf{0 , 8 9}$ & $\mathbf{0 , 8 1}$ & $\mathbf{- 0 , 6 9}$ & $\mathbf{- 0 , 8 6}$ \\
\hline $\mathrm{U}$ & 0,12 & $\mathbf{0 , 6 8}$ & $-0,10$ & $-0,05$ & $\mathbf{- 0 , 4 3}$ & $-0,25$ & $\mathbf{0 , 7 1}$ & 0,21 & $\mathbf{- 0 , 4 6}$ & $-0,31$ \\
\hline $\mathrm{V}$ & 0,10 & $\mathbf{0 , 6 1}$ & $-0,11$ & $-0,08$ & $\mathbf{- 0 , 3 6}$ & $-0,22$ & $\mathbf{0 , 7 4}$ & 0,22 & $\mathbf{- 0 , 5 0}$ & $-0,30$ \\
\hline $\begin{array}{l}\text { Precipitación } \\
\text { máxima } \\
\text { diaria }\end{array}$ & 0,29 & $\mathbf{0 , 6 0}$ & $-0,27$ & 0,07 & $-0,15$ & $-0,04$ & $\mathbf{0 , 5 9}$ & $\mathbf{0 , 5 0}$ & $\mathbf{- 0 , 6 5}$ & $\mathbf{- 0 , 6 3}$ \\
\hline $\begin{array}{l}\text { Precipitación } \\
\text { total }\end{array}$ & 0,19 & $\mathbf{0 , 5 0}$ & $-0,14$ & 0,17 & $-0,14$ & 0,20 & $\mathbf{0 , 6 9}$ & 0,36 & $\mathbf{- 0 , 5 6}$ & $-0,45$ \\
\hline
\end{tabular}

Table III: Coeficientes de correlación lineal (r) de las series de CO2 global anual y O3 promedio polar (entre 60 y $90^{\circ} \mathrm{S}$ ) de Septiembre con las series de precipitación de Tucumán y las variables $\mathrm{U}, \mathrm{V}, \mathrm{P}$ y T con promedio móvil de 5 años. En negrita se marcan los coeficientes significativos al $95 \%$ según el test t de Student.

Barreiro et al. (2014) mostraron que la combinación de un Océano Atlántico ecuatorial con anomalías frías y un Océano Pacifico ecuatorial con anomalías cálidas estuvo asociada al marcado incremento en las precipitaciones en el norte de Argentina en la década de 1970. La transición de la temperatura del Atlántico alrededor de la década del '70 favoreció el incremento de la precipitación y a partir de la década del ' 80 el cambio de fase de la PDO ayudó a mantener las anomalías positivas a partir de entonces. El mecanismo físico propuesto en su trabajo consiste en un aumento del flujo de humedad del Atlántico al Amazonas inducido por un Pacífico ecuatorial más cálido y un Atlántico ecuatorial más frio. Este flujo de humedad es desviado debido a los Andes, y en combinación con un anticiclón en niveles altos centrado en $40^{\circ} \mathrm{S}-40^{\circ} \mathrm{O}$ favorece la convergencia de humedad en el norte de Argentina. Nuestros resultados muestran que desde 1950 la precipitación presenta a grandes rasgos variaciones similares a la PDO, aunque previo a esto las series prácticamente no muestran co-variabilidad.

Análogamente, se calculó la correlación entre la PDO y las variables U, V, P y T. Al igual que con la precipitación, en 1911-2012 ninguna de dichas variables tiene correlación significativa con PDO (Tabla III). En tanto, en 1960-2012 se obtuvo correlación significativa para PDO solo con $\mathrm{U}(\mathrm{r}=0,68)$ y $\mathrm{V}(\mathrm{r}=0,61)$. De esta manera, la influencia de la PDO en la circulación podría ser en el viento zonal y meridional de la zona continental, aunque con importancia solo desde la segunda mitad del siglo XX(Figura 6d). Esto podría estar relacionado a los cambios en la circulación inducidos por la PDO, tales como el anticiclón en niveles altos centrado en $40^{\circ} \mathrm{S} 40^{\circ} \mathrm{O}$ mencionado en el trabajo de Barreiro et al. (2014) que favorecen la convergencia de humedad en el norte de Argentina y afectan a la precipitación.

La combinación de un índice AMO negativo con la PDO positiva se da antes de 1920 y posterior a 1970, coincidiendo con los mayores valores de precipitación. Esta combinación podría favorecer un Pacifico ecuatorial más cálido y un Atlántico ecuatorial más frio. Esto podría explicar que el coeficiente de correlación entre la precipitación y PDO sea significativo en la segunda mitad del siglo XX y no en el periodo 1911-2012. Así, el argumento de Barreiro et al. (2014) sobre la influencia del Atlántico-Pacifico explicaría parte del comportamiento de la precipitación en Tucumán. El salto de la precipitación total 


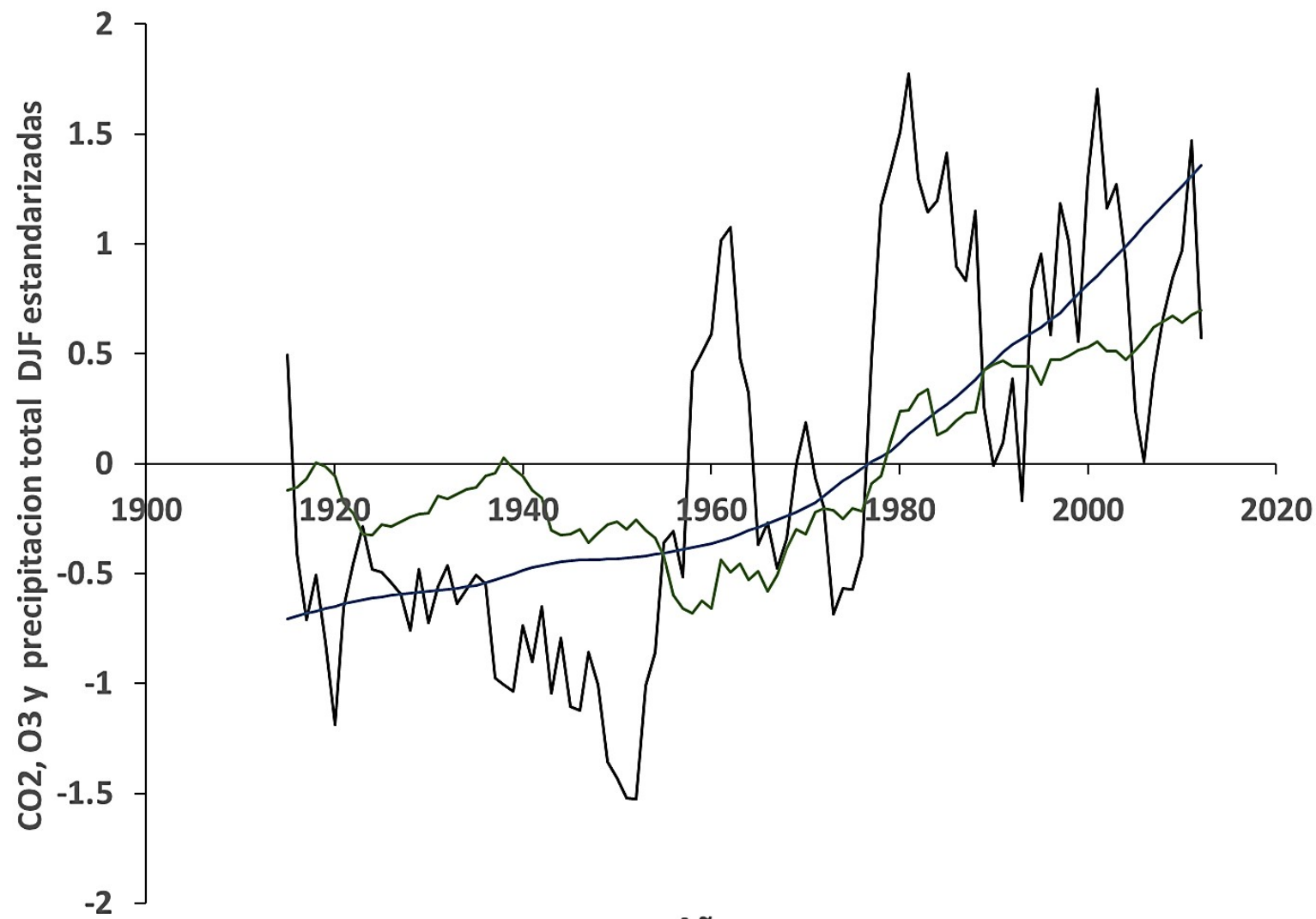

Figure 7: Series estandarizadas y suavizadas con promedio móvil de 5 años de: precipitación total de DJF de Tucumán (línea negra), CO2 global anual (lineal azul) y columna total de $\mathrm{O}_{3}$ polar de septiembre (multiplicada por -1 para una "visión directa", línea verde).

en 1956 no sería explicable con este mecanismo.

En cuanto a SOI, su coeficiente de correlación con las series de precipitación no supera el valor 0,20 en los dos periodos considerados. Esto coincide con el resultado del análisis de Minetti y Leiva (2005), quienes muestran que la Oscilación del Sur tiene débiles efectos en Tucumán. En el largo plazo, la Oscilación del Sur podría no tener influencia significativa, mientras el rol del Pacifico podría estar relacionado a PDO en combinación con AMO.

\subsection{Asociación con el dióxido de carbono y el ozono}

Las $\begin{array}{lllllll} & \text { Figuras } & 7 & \text { y } & 8 & \text { muestran las } & \text { series }\end{array}$ estandarizadas de $\mathrm{CO}_{2}$ y $\mathrm{O}_{3}$ polar junto con la precipitación total y la máxima diaria, respectivamente. Los coeficientes de correlación lineal del $\mathrm{CO}_{2}$ y $\mathrm{O}_{3}$ polar con la precipitación, U, V, P y T se listan en la Tabla III.

El $\mathrm{CO}_{2}$ presenta una variación monótona creciente, casi lineal, que es también la tendencia general de todas las variables analizadas, excepto al final de la serie, donde las variables se estabilizan o disminuyen sus valores. Esto hace que haya una correlación significativa en casi todos los casos en el periodo 1911-2012.

En el caso del $\mathrm{O}_{3}$, donde se observa una correlación negativa, los valores son altos porque el $\mathrm{O}_{3}$ presenta un cambio de tendencia general en 1960 que coincide con el cambio de tendencia en las series de precipitación y con la estabilización en los últimos años. 


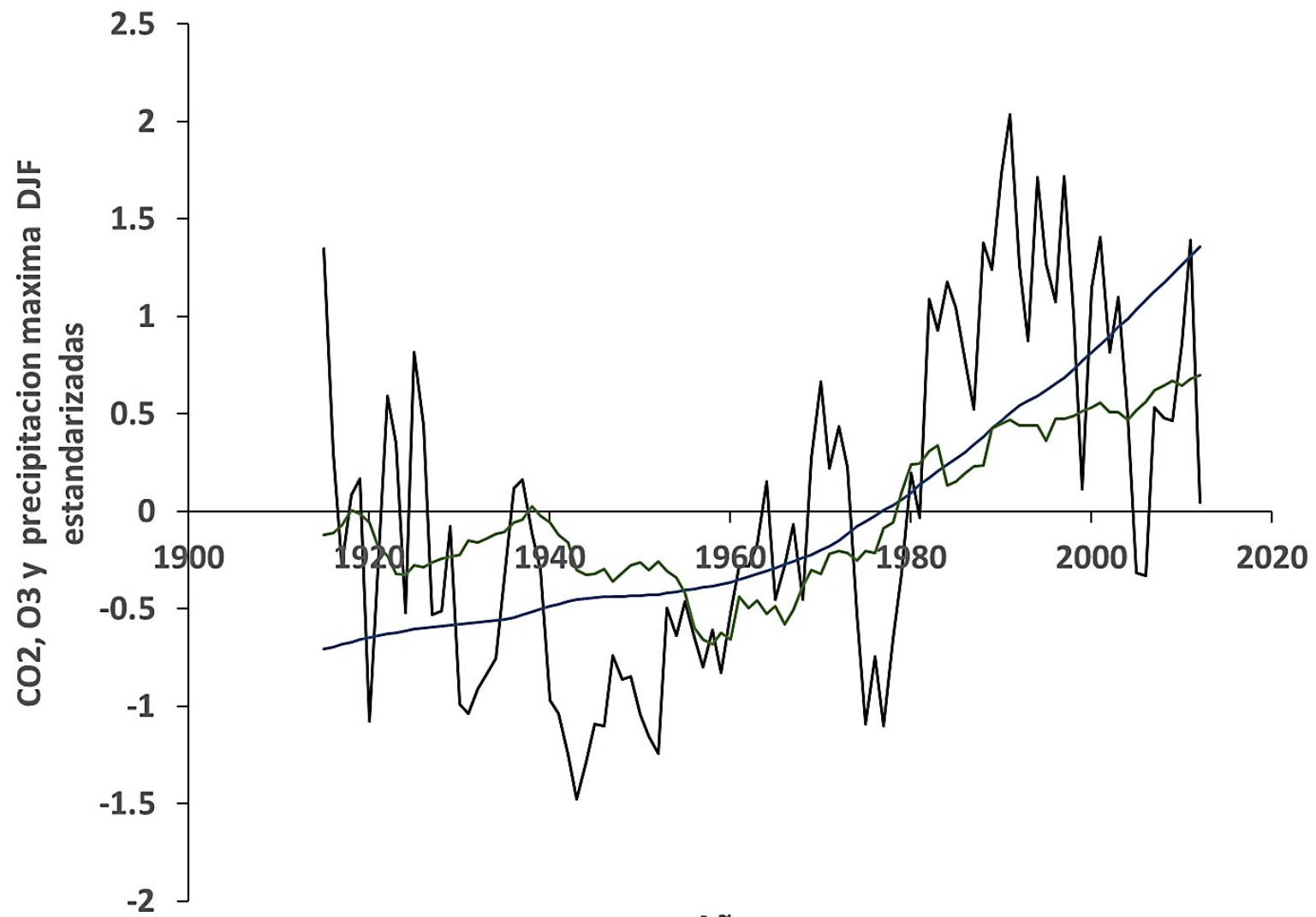

Años

Figure 8: Series estandarizadas y suavizadas con promedio móvil de 5 años de: precipitación máxima diaria de DJF de Tucumán (línea negra), $\mathrm{CO}_{2}$ global anual (línea azul) y columna total de $\mathrm{O}_{3}$ polar de septiembre (multiplicada por -1 para una "visión directa", línea verde).

Si analizamos solo el sub-periodo 1960-2012, la correlación de las series es más alta con el $\mathrm{O}_{3}$ que con el $\mathrm{CO}_{2}$ en todos los casos. Esto es debido a que, como se mencionó, además de tener una tendencia monótona la serie el $\mathrm{O}_{3}$ al final se estabiliza y esto coincide con tendencia menos marcada o casi nula en las series de precipitación.

Las notables cuasi-periodicidades interdecadales de las series de $\mathrm{P}$, de $\mathrm{U}$ y de $\mathrm{V}$ hace que la correlación en estos casos sea menor, ya que son oscilaciones totalmente ausentes en el $\mathrm{CO}_{2}$ y débiles en el $\mathrm{O}_{3}$. En cambio, en $\mathrm{T}$ predomina tendencia monótona, lo que provoca como resultado una alta correlación (mayor a $0,8)$ con las series de $\mathrm{CO}_{2}$ y $\mathrm{O}_{3}$; esto concuerda con el aumento de temperatura global de origen antropogénico.

\section{DISCUSIÓN Y CONCLUSIONES}

La precipitación total de DJF en Tucumán presenta los mayores coeficientes de correlación con $\mathrm{T}(r=0,76)$ y con $\mathrm{P}(r=0,70)$ cuando se considera el período 1911-2012. Para el sub-período 1960-2012 la correlación con P casi no cambia $(r=0,72)$ y con $\mathrm{T}$ disminuye $(r=0,44)$. Esto se puede observar en la Figura 3 , donde se ve claramente que en el sub-período, si bien hay una tendencia similar a la de $\mathrm{T}$, es mayor la cuasi-periodicidad de 20 años que acompaña a $\mathrm{P}$.

Para evaluar estadísticamente la cuasi-periodicidad mencionada, se calculó la transformada de wavelet (TW), la coherencia de wavelet (CW) y el espectro cruzado de wavelet $(\mathrm{ECW})$ entre $\mathrm{P}$ y la precipitación 

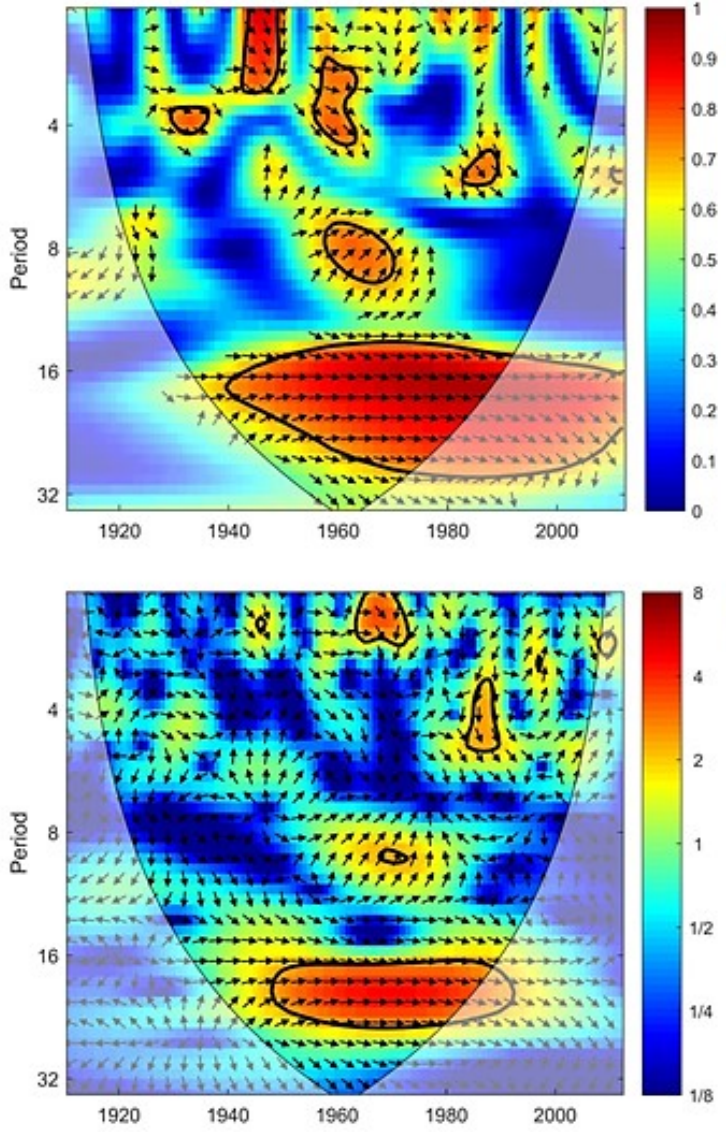

Figure 9: (a) Coherencia de wavelet y (b) espectro cruzado de wavelet entre $\mathrm{P}$ y la precipitación total de verano.

total (ambas sin promedio móvil). TW, a diferencia del espectro de Fourier, es una técnica capaz de detectar periodicidades variables en el tiempo. CW mide las oscilaciones comunes en ambas series y ECW mide la correlación entre estas oscilaciones, ambos en términos de frecuencia. Seguimos el mismo procedimiento de Heredia et al. (2019), utilizando el algoritmo desarrollado por Torrence y Compo (1998) y el software desarrollado por AslakGrinsted (disponible en http: //www.glaciology.net/wavelet-coherence/) (Grinsted et al., 2004). Se observa una clara periodicidad común y en fase de 20 años a partir de 1960 (Figura 9b) con una correlación mayor a 0,8 (Figura 9a), con un $95 \%$ de nivel de confianza. Por lo tanto, la precipitación muestra variaciones periódicas de 20 años presentes en $\mathrm{P}$, y el forzante detrás de estos cambios podría responder a la dinámica del movimiento del baricentro solar, aunque el mecanismo subyacente que sustentaría el buen acuerdo estadístico hallado es aún desconocido.

En cuanto a la buena correlación obtenida con T, la misma es fuertemente dependiente del periodo analizado, como se observa en los coeficientes de correlación presentados anteriormente. La buena correlación podría deberse a que ambas presentan tendencia lineal positiva y no a que haya una conexión física real. La tendencia lineal en $\mathrm{T}$ desde la segunda mitad del siglo XX es muy similar a la tendencia de $\mathrm{CO}_{2}(\mathrm{r}=0,89)$ y posiblemente debida al calentamiento global de las últimas décadas.

En el caso de la precipitación máxima diaria la mayor correlación se observa con las series de $\mathrm{T}$, $\mathrm{U}$ y $\mathrm{V}$, con valores similares (de 0,5 a 0,6$)$ tanto el periodo completo como en el sub-periodo.

Lo expuesto anteriormente está de acuerdo con el efecto de las condiciones del Atlántico sobre la precipitación en el NOA y también con el del sistema cuasi-monzónico en la región norte de Argentina, Bolivia y Brasil. Específicamente, los parámetros de regiones oceánicas analizados $(\mathrm{P}$ y $\mathrm{T}$ ) muestran mayor correlación con la precipitación total, mientras que los parámetros continentales ( $\mathrm{U}$ y $\mathrm{V}$ ) adquieren importancia al estudiar la máxima diaria.

En cuanto al impacto de la variabilidad oceánica, las series de precipitación parecen estar moduladas por la PDO en combinación con AMO desde la década del 70, variaciones que también se observan en las series de U y V. Por otra parte, el corrimiento en la época en que suele ocurrir el máximo diario anual, mencionado en la Sección 4-A,podría ser indicador de un aumento en la duración del periodo de lluvias. A su vez, esto podría estar relacionado al aumento de la duración del monzón sudamericano desde 1971 documentado por Carvalho et al. (2011), quienes lo asocian a la transición climática de la década del 70 relacionada a la PDO. Así, la PDO junto 


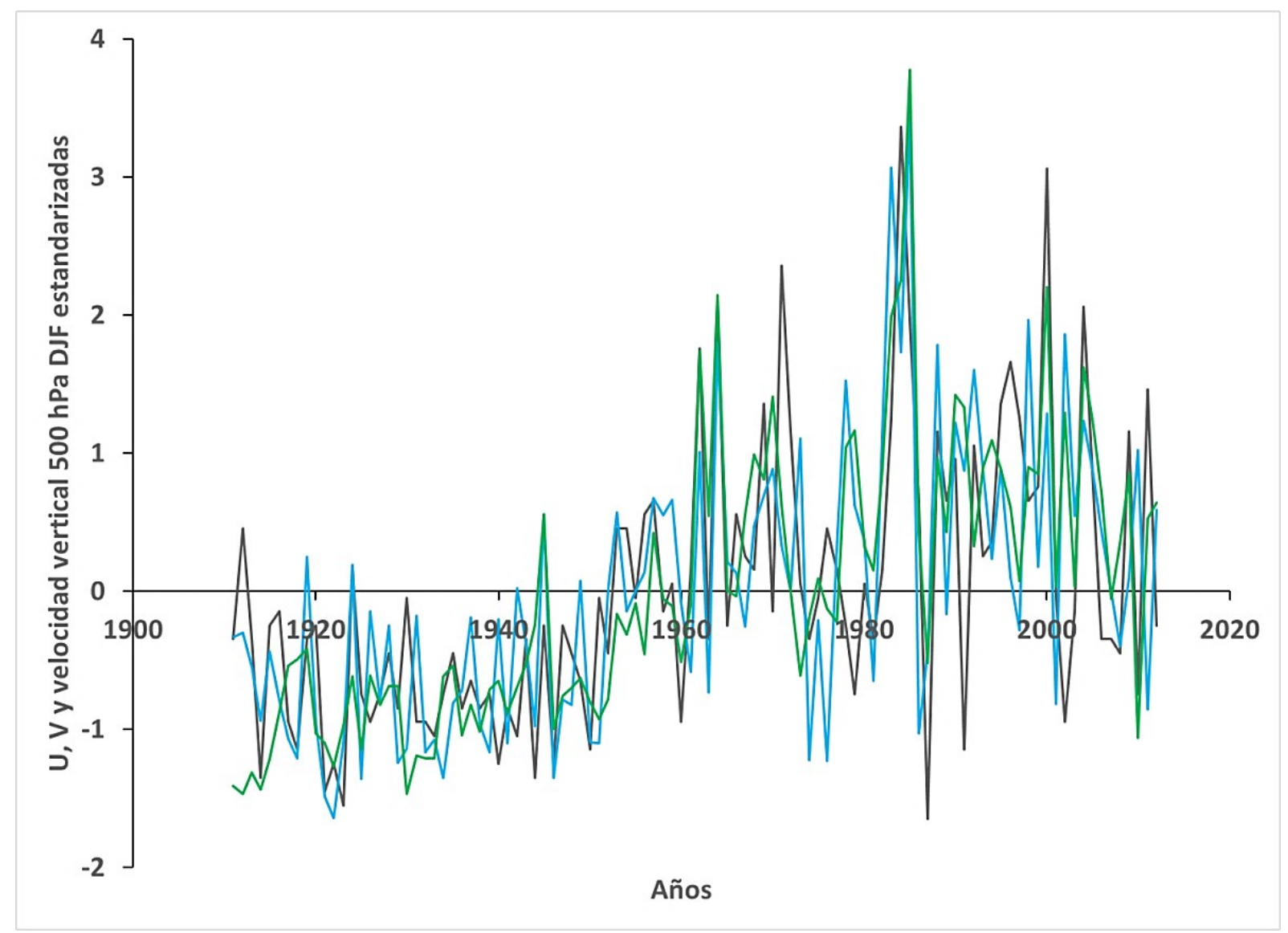

Figure 10: Series estandarizadas de: U promedio del área comprendida entre $15^{\circ} \mathrm{S}-25^{\circ} \mathrm{S}$ y $60^{\circ} \mathrm{O}-90^{\circ} \mathrm{O}$ (línea celeste), $\mathrm{V}$ promedio del área comprendida entre $15^{\circ} \mathrm{S}-25^{\circ} \mathrm{S}$ y $50^{\circ} \mathrm{O}-70^{\circ} \mathrm{O}$ (línea verde) y velocidad vertical en $500 \mathrm{hPa}$ de la misma región que U (línea gris).

con la AMO tendrían un rol importante sobre la precipitación en Tucumán.

Respecto al aumento del $\mathrm{CO}_{2}$, además del aumento de la temperatura global, provocaría un aumento de las precipitaciones debido a la intensificación del ciclo hidrológico. En este sentido, Vera y Díaz (2014) encontraron que los modelos que consideran el aumento de gases de efecto invernadero como único forzante son capaces de reproducir sólo parcialmente las tendencias en la región a la que pertenece Tucumán. Nuestros resultados estadísticos sugieren que el $\mathrm{O}_{3}$ y el movimiento de baricentro solar podrían también impactar en el comportamiento a largo plazo de la precipitación, aunque un análisis más exhaustivo es necesario para fundamentar esto.
En cuanto al $\mathrm{O}_{3}$, las correlaciones negativas encontradas estarían de acuerdo con los resultados de Kang et al. (2011) y Gonzalez et al. (2014) quienes señalan que una disminución de ozono polar induciría un aumento en la precipitación en regiones de Sudamérica. Precisamente, Cai y Cowen (2007) remarcaron la importancia de contar con modelos que cuantifiquen la contribución del $\mathrm{O}_{3}$, debido a que se proyecta que el $\mathrm{O}_{3}$ estará completamente recuperado hacia el 2050, y su impacto en el clima del hemisferio sur sería opuesto al del incremento del $\mathrm{CO}_{2}$.

Respecto a las variables de circulación, en la Figura 10 se muestran las series estandarizadas de $\mathrm{U}, \mathrm{V}$ y velocidad vertical en $500 \mathrm{hPa}$ (indicador de circulación vertical en la troposfera 
media) en la región en la que se analizó U, obtenido también del reanalisis $20 \mathrm{CR}$ V2c. Se observa buen acuerdo entre dichas series, con valores de correlación con la velocidad vertical de 0,6 con U y 0,7 con V (sin suavizado). Esto indica que las variaciones de U están asociadas a variaciones en la actividad convectiva en esta región perteneciente al Alta de Bolivia. Por otro lado, se dio una disminución en el flujo meridional de vientos en capas bajas (V). Así, los cambios en la precipitación máxima diaria, que presenta buena correlación con U y V, no estarían asociados a cambios en la advección meridional de vapor mediante el jet de niveles bajos ( $\mathrm{V}$ disminuyó), sino más bien a las variaciones en la convección. El aumento de la convección en la parte subtropical de Sudamérica en la segunda mitad del siglo XX fue reportado por Saurral et al. (2017), en concordancia con el presente análisis. El mecanismo físico detrás de la alta correlación entre $\mathrm{U}$ y el $\mathrm{CO}_{2}$ podría consistir en un aumento en la actividad convectiva inducida por el aumento de la temperatura global. Chen et al. (2020) encuentran un aumento generalizado a nivel global en el valor medio de la energía potencial convectiva disponible (CAPE, Convective Available Potential Energy) en DJF como consecuencia del aumento de $\mathrm{CO}_{2}$. En el centro y norte de Argentina el mayor aumento del CAPE se observaría en el Noroeste Argentino. Ellos postulan que el aumento del CAPE incrementaría la probabilidad de ocurrencia de eventos de convección profunda, de tal manera que en nuestra región podríamos esperar un aumento en la precipitación máxima diaria ante el aumento de $\mathrm{CO}_{2}$. Desde ya que es necesaria una investigación más profunda y detallada para resolver la relación entre el aumento del CAPE y su posible efecto en la precipitación.

En la Tabla III se observa que la correlación de $\mathrm{U}$ y V con el $\mathrm{CO}_{2}$ baja notoriamente cuando se considera el sub-período 1960-2012. Esto se debe a que, como se observa claramente de la Figura 10, en ese período U y V prácticamente no presentan tendencia lineal neta.

$\mathrm{Al}$ no presentar una tendencia sostenida $\mathrm{y}$ además al tener una marcada cuasi-periodicidad, $\mathrm{P}$ presenta una notable menor correlación con $\mathrm{CO}_{2}$ que las demás variables. En el hemisferio sur la disminución de $\mathrm{O}_{3}$ y el aumento de $\mathrm{CO}_{2}$ del siglo pasado aportaron a las tendencias de expansión de la Celda de Hadley y produjeron un aumento de los valores de presión de los anticiclones subtropicales en la segunda mitad del siglo XX (Lucas et al. 2014, Nguyen et al. 2015). La estabilización del $\mathrm{O}_{3}$ de los últimos años de la serie coincide con la ausencia de tendencia lineal en la serie de precipitación total y de $\mathrm{P}$ en el Atlántico. Al estar proyectada una recuperación del ozono en las próximas décadas, una posibilidad es que los valores de precipitación total de DJF en Tucumán disminuyan o se estabilicen, tal como proponen Gonzalez et al. (2014) para el sudeste de Sudamérica. Sin embargo, el aumento de $\mathrm{CO}_{2}$ también produce tendencias de aumento en la precipitación, por lo que una vez recuperado el $\mathrm{O}_{3}$ aún podría haber un nuevo aumento de las precipitaciones posterior a la recuperación del $\mathrm{O}_{3}$.

Es importante destacar que la serie de precipitación de Tucumán tiene gran variabilidad interanual e interdecadal. En particular la variación bidecadal en la precipitación total a partir de 1960 y en $\mathrm{P}$ en el período completo,que está presente también en la posición del baricentro solar.Hay otras variaciones en la precipitación total, y en especial en la máxima diaria, $\mathrm{U}$, y $\mathrm{V}$ que podrían estar relacionadas a la PDO. Si bien se detectaron correlaciones altas con las variables mencionadas, se necesita un "sustento físico"para el análisis estadístico, en especial en relación a la influencia del baricentro. Por otra parte, se debería evaluar el efecto (refuerzo o atenuación) que los forzantes antropogénicos podrían tener en PDO y AMO considerando que estas oscilaciones parecen tener fuerte influencia en la precipitación. La profundización en el estudio de los mecanismos subyacentes podría ser clave para mejores proyecciones de la precipitación a largo plazo en escala local. 
Agradecimientos: Los autores agradecen a la Estación Experimental Agroindustrial Obispo Colombres (EEAOC) por los datos cedidos y a los dos revisores anónimos por sus valiosos aportes a este artículo. Franco D. Medina y Flavia M. Bazzano agradecen al CONICET por sus respectivas becas de investigación. Parte de este trabajo fue financiado con los Proyectos PICT 2015-0511 y PIUNT E642.

\section{REFERENCIAS}

Barreiro, M., Diaz, N., Renom, M., 2014: Role of the global oceans and land-atmosphere interaction on summertime interdecadal variability over northern Argentina. Climate Dynamics, 42, 1733-1753. https://doi.org/ 10.1007/s00382-014-2088-6.

Barros, V., Castañeda M.E., Doyle M., 2000a: Recent Precipitation Trends in Southern South America East of the Andes. An Indication of Climatic Variability. In. Smolka P., Volkheimer W. (eds) Southern Hemisphere Paleo- and Neoclimates. Springer, Berlin, Heidelberg. https://doi.org/10. 1007/978-3-642-59694-0_13.

Barros, V., Gonzalez, M., Liebmann, B., Camilloni, I.,2000b: Influence of the South Atlantic convergence zone and SouthAtlantic Sea surface temperature on interannualsummerrainfall variability in Southeastern South America. TheorApplClimatol 67, 123-133, https: //doi.org/10.1007/s007040070002

Bazzano, F., Heredia, T., Elías, A. G., Lamelas, C.,Forciniti, J., 2019: Tendencias en la Precipitación en Tucumán bajo Efecto del Cambio Climático. RevistaMeteorológica. 44, 2, 1-14.

Bettolli, M.L., Penalba, O.C., 2018: Statistical downscaling of daily precipitation and temperatures in southern La Plata Basin. Int J Climatol. 38, 3705- 3722. https://doi org/10.1002/joc.5531.

Biasutti, M., 2013: Climate change, future rise in rain inequality, Nature Geoscience 6, 337-338.

Cai, W., Cowan, T., 2007: Trends in Southern Hemisphere Circulation in IPCC AR4 Models over 1950-99. Ozone Depletion versus Greenhouse Forcing J. Climate, 20,681-693, https://doi.org/10.1175/JCLI4028.1.

Campetella, C. M., Vera, C., 2002: The influence of the Andes mountains on the South American low-level flow, Geophys. Res. Lett., 29,17, 1826, https://doi.org/ 10.1029/2002GL015451.

Carvalho, L.M.V., Jones, C., Silva, A.E., Liebmann, B. and Silva Dias, P.L., 2011: The South American Monsoon System and the 1970s climate transition. Int. J. Climatol., 31: 1248-1256. doi:10.1002/joc. 2147

Chen, T. S., Weng, S., Schubert, S., 1999: Maintenance of Austral Summertime Upper-Tropospheric Circulation over Tropical South America. The Bolivian High-Nordeste Low System. J. Atmos. Sci., 56, 2081-2100, https://doi.org/ 10.1175/1520-0469(1999) 056<2081: MOASUT $>2.0 . \mathrm{CO} ; 2$

Chen, J., Dai, A., Zhang, Y., Rasmussen, K.L., 2020: Changes in Convective Available Potential Energy and Convective Inhibition under Global Warming. J. Climate, 33, 2025-2050, https: //doi.org/10.1175/JCLI-D-19-0461.1.

Cionco, R.G.,Pavlov, D.A., 2018: Solar barycentric dynamics from a new solar-planetary ephemeris. Astron. Astrophys. 615, A153. https://doi.org/10.1051/ 0004-6361/201732349

Compo, G. P., Whitaker, J. S.,Sardeshmukh, P. D., Matsui, N., Allan, R. J., Yin, X., et al., 2011: The Twentieth Century Reanalysis Project. Quarterly J. Roy. Meteorol. Soc., 137, 1-28. https://doi.org/10.1002/qj.776.

Ferrero, M.A., Villalba R., 2019:Interannual and Long-Term Precipitation Variability Along the Subtropical Mountains and Adjacent Chaco ,22-29을 S: in Argentina, Front. Earth Sci., 7, 148. https://doi.org/10.3389/ feart.2019.00148.

Gan, M.A., Rao, V.B.,Moscati, M.C.L., 2005: South American monsoon indices. Atmosph. Sci. Lett., 6. 219-223. https://doi.org/10. 1002/asl.119.

Gonzalez, P.L.M., Polvani, L.M., Seager, 
R., 2014: Stratospheric ozone depletion. a key driver of recent precipitation trends in South Eastern South America ClimDyn: 42, 1775. https: //doi.org/10.1007/s00382-013-1777-x.

Grinsted, A., Moore, J., Jevrejeva, S., 2004:Application of the cross wavelet transform and wavelet coherence to geophysical time series. Nonlinear Process Geophys. 11, 561-566. https: //doi.org/10.5194/npg-11-561-2004.

Hamed, K.H, 2008: Trend detection in hydrologic data. The Mann-Kendall trend test under the scaling hypothesis. J. Hydrol. 349, 350363 , https://doi.org/10.1016/j.jhydrol 2007.11.009.

Heredia T., Bazzano F. M., Cionco R.G., Soon W, Medina F. D., Elias A. G., 2019:Searching for solar-like interannual to bidecadal effects on temperature and precipitation over a Southern Hemisphere location. Journal of Atmospheric and Solar-Terrestrial Physics, Volume 193, 2019, 105094, ISSN 1364-6826, https://doi.org/ 10.1016/j.jastp.2019.105094.

Kang S. M., Polvani L. M., Fyfe J. C., Sigmond M., 2011: Impact of Polar Ozone Depletion on Subtropical Precipitation. Science. 332, 6032, 951-954. https://doi.org/10.1126/ science.1202131.

Labraga, J.C., 2010: Statistical downscaling estimation of recent rainfall trends in the eastern slope of the Andes mountain range in Argentina. TheorApplClimatol 99, 287-302. https://doi.org/10.1007/ s00704-009-0145-6.

Lucas C., Timbal B., Nguyen, H., 2014: The expanding tropics. a critical assessment of the observational and modeling studies. WIREs Clim Change, 5. 89-112. https://doi.org/ 10.1002/wcc. 251

Marengo, J.A., Soares, W.R., Saulo, C., Nicolini, M., 2004: Climatology of the Low-LevelJet East of the Andes as Derived from the NCEP-NCAR Reanalyses. Characteristics and Temporal Variability. J. Climate, 17, 2261-2280. https://doi.org/10.1175/1520-0442,
2004:017<2261.COTLJE >2.0.CO;2.

Medina, E. R. y. Minetti, J. L., 2004: El salto climático y su impacto en la frecuencia de precipitaciones máximas diarias en San Miguel de Tucumán. X Reunión y LV Latinoamericana de Agrometeorología. Mar del Plata, Argentina.

Meehl, G.A., Stocker, T. F., Collins, W. D., Friedlingstein, P., Gaye, T., Gregory, J. M., et al., 2007:Global Climate Projections.In Climate Change 2007: The Physical Science Basis. Contribution of Working Group I to the Fourth Assessment Report of the Intergovernmental Panel on Climate Change, Cambridge University Press, pp 747-846

Minetti, J.L., 1999: Las sequías en la Argentina. Contribuciones Científicas GAEA. 60va. Sem. De Geografía., 491-96. San Juan.

Minetti, J. L., Leiva, M. del V., 2005: Variabilidad y Cambio Climático en el Noroeste Argentino. Cap. 12 en El Clima en el NOA. 410 páginas. ISBN: 987-9390-66-0. Edit. Magna. S Miguel de Tucumán. Tucumán. Argentina.

Minetti, J. L.; Vargas, W. M., 1997: Trends and jumps in the annual precipitation in South America, south of the $15^{\circ}$ S.Atmósfera, ISSN 0187-6236, 11, 4, 205-221

Minetti, J.L., Vargas, W.M., Poblete, A.G., Acuña, L.R., Casagrande, G., 2003: Non-linear trends and low frequency oscillations in annual precipitation over Argentina and Chile, 1931-1999, Atmósfera, 16, 119-135.

Nguyen H., Lucas., Evans A., Timbal B., Hanson L.,2015: Expansion of the Southern Hemisphere Hadley Cell in Response to Greenhouse Gas Forcing. J. Climate, 28, 8067-8077. https: //doi.org/10.1175/JCLI-D-15-0139.1.

NicoliniM, Saulo A., Torres J., Salio P., 2002: Enhanced precipitation over Southeastern South America related to strong low-level jet events during austral warm season. Meteorologica, Special Issue for the South American Monsoon System. 27.

Palomino Lemus, R., Córdoba Machado, S, Gámiz Fortis, S. R., Castro-Díez, Y., 
Esteban Parra, M. J., 2017: Climate change projections of boreal summer precipitation over tropical America by using statistical downscaling from CMIP5 models Environ. Res. Lett.12 124011. https://doi.org/10 1088/1748-9326/aa9bf7.

Pettitt A. N., 1979: A non-parametric approach to the change-point problema. Appl. Stat. 28. 126-135

Polvani, L.M., Waugh, D.W., Correa, G.J., Son S., 2011: Stratospheric Ozone Depletion: The Main Driver of Twentieth-Century Atmospheric Circulation Changes in the Southern Hemisphere. J. Climate, 24, 795-812, https: //doi.org/10.1175/2010JCLI3772.1.

R Core Team, 2019: R. A language and environment for statistical computing. RFoundation for Statistical Computing, Vienna, Austria. https://www.R-project org/.

Saurral, R. I., Camilloni, I. A., Barros, V. R., 2017: Low-frequency variability and trends in centennial precipitation stations in southern South America. Int. J. Climatol., 37. 1774-1793. https://doi.org/10.1002/ joc. 4810 .

Sen, P.K., 1968: Estimates of the regression coefficient based on Kendall's tau. J. Am. Statist. Assoc. 63, 1379-1389.

Thorsten Pohlert, 2018: Trend. Non-Parametric Trend Tests and Change-PointDetection. $\mathrm{R}$ package version 1.1.1. https://CRAN R-project.org/package=trend

Torrence, C., Compo, G.P., 1998: A practical guide to wavelet analysis. Bull. Am. Met. Soc. 79, 61-78. https: //doi.org/10.1175/1520-0477,1998: 079<0061:APGTWA>2.0.C0;2.

Trouet, V., Van Oldenborgh, G. J., 2013: KNMI Climate Explorer. a web-based research tool for high-resolution paleoclimatology, Tree-Ring Research, 69, 3-14. https://doi. org/10.3959/1536-1098-69.1.3.

Van Oldenborgh, G.J., Coelho, C.A.S., Doblas-Reyes, F. J., 2008: Exploratory analysis and verification of seasonal forecasts with the KNMI climate explorer, ECMWF Newsletter 116, 4-5. ,Avalible at https://www.ecmwf.int/ sites/default/files/elibrary/2008/ 14609-newsletter-no116-summer-2008. pdf:

Vera C.,Díaz, L., 2015: Anthropogenic influence on summer precipitation trends over South America in CMIP5 models. Int. J. Climatol., 35. 3172-3177. https://doi.org/10.1002/ joc. 4153

Vera C., Higgins, W., Amador, J., Ambrizzi, T., Garreaud, R. D., Gochis, D., et al., 2006: Toward a unified view of the American monsoon systems. J. Clim. 19, 4977-5000. https://doi.org/10.1175/jcli3896.1.

Vera C., Díaz L., Saurral R., 2019: Influence of Anthropogenically-Forced Global Warming and Natural Climate Variability in the Rainfall Changes Observed Over the South American Altiplano. Front. Environ. Sci. 7:87. https://10.3389/fenvs.2019.00087.

Este es un artículo de acceso abierto distribuido bajo la licencia Creative Commons, que permite el uso ilimitado, distribución y reproducción en cualquier medio, siempre que la obra original sea debidamente citada. 